\title{
Caractérisation didactique des gestes de l'aide ordinaire à l'école élémentaire : une étude comparative de deux cas didactiques limite en mathématiques
}

A characterization of actions carried out in ordinary pedagogical aid at primary school : a comparative study of two borderline cases in maths

Corinne Marlot et Marie Toullec-Théry

\section{OpenEdition}

Journals

Édition électronique

URL : http://journals.openedition.org/educationdidactique/1081

DOI : 10.4000/educationdidactique.1081

ISSN : 2111-4838

Éditeur

Presses universitaires de Rennes

Édition imprimée

Date de publication : 30 décembre 2011

Pagination : 7-32

ISBN : 978-2-7535-1832-2

ISSN : 1956-3485

Référence électronique

Corinne Marlot et Marie Toullec-Théry, « Caractérisation didactique des gestes de l'aide ordinaire à l'école élémentaire : une étude comparative de deux cas didactiques limite en mathématiques », Éducation et didactique [En ligne], 5-3 | 2011, mis en ligne le 30 décembre 2013, consulté le 09 décembre 2020. URL : http://journals.openedition.org/educationdidactique/1081 ; DOI : https:// doi.org/10.4000/educationdidactique.1081

Ce document a été généré automatiquement le 9 décembre 2020

Tous droits réservés 


\section{Caractérisation didactique des gestes de l'aide ordinaire à l'école élémentaire : une étude comparative de deux cas didactiques limite en mathématiques}

A characterization of actions carried out in ordinary pedagogical aid at primary school: a comparative study of two borderline cases in maths

Corinne Marlot et Marie Toullec-Théry

\section{Introduction}

1 Notre approche prend son origine dans l'approche comparatiste (Mercier, SchubauerLéoni \& Sensevy, 2002). Dans cet article, nous comparons l'action didactique de deux professeurs, lors de situations d'aide « ordinaire » en mathématiques en classe de CE2. Notre ambition est de comprendre, au sens de "prendre ensemble ", ce qui va orienter les apprentissages effectifs dans la classe. Il va s'agir, au cours de ce travail, de clarifier ce que pourraient être certaines des déterminations de l'action conjointe quand le professeur aide des élèves dans le cours ordinaire de la classe.

2 Un des enjeux de la théorie de l'action conjointe en didactique (Sensevy \& Mercier, 2007) - que nous nommerons ensuite TACD - est de rendre compte de la dynamique des transactions ${ }^{1}$ entre le professeur et les élèves, le savoir tenant lieu d'objet transactionnel. Dans cette théorie, la notion de Jeu est une métaphore de l'action conjointe. Le Jeu y est considéré comme une pratique soumise à des contraintes et à des règles (Chauvier, 2007). L'usage de cette métaphore ouvre aux notions connexes 
d'enjeux et de gain; le gain étant ici synonyme de profit symbolique. Dans le contexte des situations didactiques, il y a gain quand les attentes professorales sont satisfaites. Du point de vue du chercheur, ces attentes peuvent cependant relever d'une différence de degré ou d'une différence de nature. En terme de « degré », le professeur peut avoir un niveau d'exigence plus ou moins important. En terme de "nature", le professeur peut assimiler "faire apprendre» à "faire $\operatorname{réussir}^{2}$ »: le gain n'est pas toujours directement connecté à l'apprentissage effectif. La preuve en est que, pour l'élève, une manière de satisfaire aux exigences de l'enseignant se résume souvent, plus qu'à apprendre, à produire le bon comportement (une réponse, une attitude, etc.) qui permettra au Jeu didactique de se poursuivre.

Outre cette notion de gain, le Jeu didactique est caractérisé par sa dimension coopérative - le professeur ne gagne que si les élèves gagnent -, mais aussi par sa dimension "négociative ». Considérer le savoir comme objet transactionnel, oblige alors à penser «le relationnel au cœur du savoir et non l'inverse» (Auriac, 2006), c'est-àdire tenter de comprendre comment le savoir structure les transactions entre les acteurs ; en fait, à quoi jouent le professeur et les élèves et en quoi ces Jeux s'actualisent dans des discours. De plus, le professeur, dans son travail habituel, produit sans cesse des actions de régulation qui lui permettent de gérer la tension entre l'avancée du temps didactique et l'hétérogénéité des élèves (Sensevy, Maurice, Clanet \& Murillo, 2008 ; Chopin, 2008, 2010).

Dans les épisodes d'aide « ordinaire » étudiés, nous allons nous attacher à comprendre comment le professeur, au cours de ses actions de régulation, réaménage progressivement le milieu de l'étude ${ }^{3}$. Cette recherche se propose d'étudier « comment vivent" les objets sélectionnés par le professeur en insistant sur les phases de régulation de l'activité des élèves en situation. Dans cette perspective, nous allons nous appuyer sur la notion de milieu, développée en didactique (Amade-Escot \& Venturini, 2009; Brousseau, 1998; Chevallard, 1992; Schubauer-Léoni \& al., 2007; Sensevy \& Mercier, 2007). Notre observation se focalisera sur la synergie qui se développe entre les intentions des professeurs et les objets du milieu.

Nos travaux précédents (Toullec-Théry, 2006; Marlot, 2008) ont montré que, dans certaines conditions, l'aménagement du milieu et parfois même les réaménagements successifs peuvent éloigner les élèves des enjeux de savoir. De fait, la situation d'enseignement-apprentissage semble migrer vers des zones à la limite du didactique. Dans ces zones " critiques ", les savoirs en jeu peuvent changer de forme ou s'évanouir. On observe alors une certaine déstabilisation du côté du professeur, comme du côté des élèves (Toullec-Théry, Marlot, 2009). Ces milieux transactionnels ${ }^{4}$ singuliers vont jouer pour nous le rôle de "loupe " parce qu'ils vont nous permettre de prendre la mesure des écarts entre les apprentissages attendus et ceux effectivement observés. Ainsi, ce travail nous permettra de produire les premières caractéristiques de ce que nous appelons un cas didactique limite.

\section{Des outils théoriques issus de la TACD}

Nous appréhendons le réaménagement du milieu de l'étude par le professeur et les élèves en appréciant les écarts entre les apprentissages attendus par le professeur et les apprentissages effectifs des élèves, dans le cadre de ces « cas didactiques limite ». Cette orientation nous amène à considérer ce qui va faire «signe» pour le professeur et 
l'amener à produire des réaménagements du milieu. À cette fin, et du point de vue de l'analyse, nous nous appuierons sur les notions d'épistémologie pratique (Sensevy, 2006 ; Marlot, 2008, 2009) et de système Jeux d'Apprentissage/Jeux Epistémiques (Sensevy, 2007 ; Marlot, 2008 ; Santini, 2009 ; Gruson, 2009).

L'épistémologie pratique du professeur oriente l'action de l'enseignant sur la base de certaines théories ${ }^{5}$ qu'il a intégrées au cours de sa formation et de son expérience professionnelle. Toutefois, cette forme d'épistémologie pratique ne préexiste pas nécessairement à la situation. Nous dirons plutôt qu'elle naît de l'action et qu'elle l'oriente : c'est la situation qui va sélectionner certains éléments de cette épistémologie (Marlot, 2008). Ainsi, selon les contextes, un même professeur peut mobiliser des épistémologies pratiques hétérogènes. L'accès, pour le chercheur, à certains éléments de l'épistémologie pratique du professeur, permet d'éclairer les réponses singulières que l'enseignant apporte quand il est placé face à des nécessités parfois conflictuelles lors de la régulation de l'activité des élèves : maintenir la communication entre pairs tout en pacifiant les relations, porter les enjeux épistémiques tout en prêtant attention aux questions de " place » et de «face » (Goffman, 1973), faire avancer le temps didactique tout en gérant l'hétérogénéité des élèves... Dans cet article, nous nous attacherons à comprendre quelles sont les épistémologies pratiques relatives à l'aide de ces deux professeurs.

Dans le cadre de la TACD, c'est la modélisation de l'action conjointe sous forme de système Jeu d'Apprentissage (JA)/Jeu Épistémique (JE) qui nous permet de conduire cette analyse selon un point de vue dynamique. Le système JA-JE est une grille de lecture à l'usage du chercheur qui permet de voir la séance comme une suite de Jeux d'Apprentissage, orientés vers un Jeu Épistémique. Ce dernier s'entend comme l'expression d'une capacité ou d'un système de capacités visés par la situation d'apprentissage. Dans notre travail, le Jeu Épistémique est inféré par le chercheur à partir de l'analyse a priori de la situation. Les Jeux d'Apprentissage, quant à eux, fonctionnent en système et représentent le " contexte » des transactions didactiques. Comme nous le verrons plus tard dans les épisodes analysés, le passage d'un Jeu d'apprentissage à un autre s'opère quand il y a modification du contrat didactique en cours et/ou changement de focalisation sur des objets du milieu. Chaque Jeu d'Apprentissage peut donc être vu comme l'expression d'un contrat didactique dans un milieu spécifique. Un récent travail (Marlot, 2009) a permis une catégorisation des Jeux d'Apprentissage selon leur densité épistémique, c'est-à-dire selon leur degré de proximité avec le savoir en jeu. Ces Jeux d'Apprentissage peuvent alors se déployer selon un gradient épistémique qui propose une répartition des Jeux selon les deux polarités générique et spécifique.

Figure 1 : Représentation du gradient généricité/spécificité des Jeux d'Apprentissage

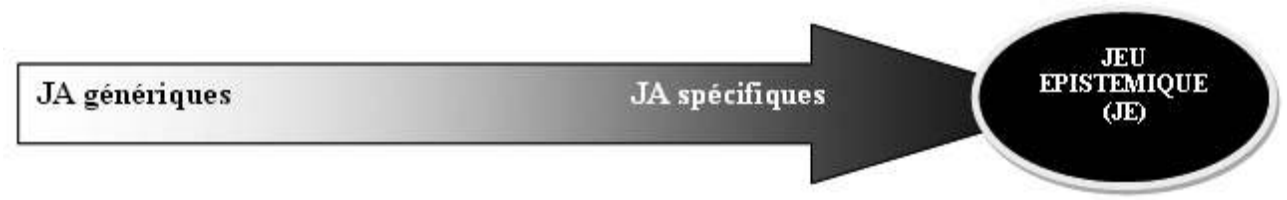

9 Toutefois, un Jeu d'Apprentissage n'est pas générique ou spécifique en lui-même et de manière définitive : cette caractéristique est attribuée de manière relative par l'analyse didactique et on peut même dire, qu'à terme, un Jeu d'Apprentissage spécifique peut 
évoluer vers un Jeu d'Apprentissage générique ${ }^{6}$. En effet, l'analyse didactique a pour rôle d'expliciter les règles qui actionnent ces Jeux d'Apprentissage et qui les font fonctionner en système. Ces règles sont de nature différente (Sensevy, 2007). Les règles définitoires des Jeux d'Apprentissage sont données par la situation et par la médiation du professeur: elles précisent "comment jouer au Jeu»; et les règles stratégiques permettent d'établir " comment gagner au Jeu » : elles représentent à la fois l'enjeu et le résultat de l'action didactique conjointe ${ }^{7}$. L'analyse didactique, en plus d'expliciter les "règles des Jeux d'Apprentissage ", va alors poursuivre une seconde visée : décrire l'évolution des objets mobilisés par la situation, afin de mieux comprendre comment se construit peu à peu le texte du savoir pour les élèves.

Enfin, les Jeux d'Apprentissage n'existent pas pour eux-mêmes : ils visent la maitrise et la construction d'un Jeu Épistémique qui représente in fine, la pratique de savoir en milieu scolaire, visée par la situation. La modélisation JA/JE engage donc le chercheur à se poser la question suivante : comment (via une succession de Jeux d'Apprentissage), le professeur et les élèves s'y prennent-ils ensemble pour gagner au Jeu, c'est-à-dire pour atteindre le Jeu Épistémique visé? S'il existe une différence de degré entre les différents types de Jeux d'Apprentissage (spécifiques et génériques), c'est une différence de nature qui existe entre les Jeux d'Apprentissage et le(s) Jeu(x) Épistémique(s).

\section{Cadre méthodologique}

Observer ce qui fait signe pour le professeur ainsi que l'évolution des objets nécessite de travailler à un grain d'analyse qui donne une place importante au «micro». C'est pourquoi, nous nous orientons vers des méthodologies fondées sur des études de cas.

12 À la suite de Bru, Altet et Blanchard-Laville (2004), nous considérons que les singularités minimes dans les pratiques des professeurs peuvent entraîner de grandes différences dans l'apprentissage des élèves. Notre travail ne portera donc pas uniquement sur la recherche des contrastes les plus prononcés, mais accordera également une place importante aux détails. Nous entrons ainsi dans la logique du « penser par cas » : «[...] La description « dense » des singularités doit s'y faire plus patiente et moins économe de ses détails ou nuances; l'invention y est peut-être plus laborieuse, pas forcément plus rare, mais d'une définition plus incertaine que dans un système fermé par les règles d'une inférence déductive ou d'une généralisation inductive » (ibid, p. 43). En accord avec Passeron et Revel (ibid, p. 29), nous disons que le cas fait preuve et que sa force " croîtra à mesure que se présenteront d'autres cas, analogues ou parents par plusieurs de leurs traits ». Établir des « collections » de cas répond à une forme de raisonnement à partir de prototype $e^{8}$. Toutefois, le système de catégories et d'inférences spécifiques produit à partir de prototypes est toujours en devenir, aussi la liste des exceptions restera-t-elle ouverte ou, si l'on préfère, nos catégories seront toujours soumises à révision. Dans cette logique de la "pensée par cas ", nous faisons le choix dès à présent de fonder notre travail sur la construction d'une première collection, celle des « cas didactiques limite».

13 Pour ce qui est de la construction des données, nous avons opéré de manière identique dans chacun des deux cas. Les données ont été collectées et analysées selon un processus d'enquête, dans le but de construire progressivement un réseau de significations. La séance filmée est toujours précédée d'un bref entretien (entretien 
ante). Le professeur peut y exprimer ses intentions, formuler ses objectifs, identifier les différents temps et les formes de travail envisagées. Après la séance, nous nous entretenons à nouveau avec le professeur (entretien post) pour savoir en quoi ce qui s'est passé est conforme ou non à ses attentes. Un troisième entretien plus conséquent, différé cette fois, est également mené : il permet à chaque professeur de s'exprimer en présence de la vidéo, sur un ou plusieurs épisodes clé repérés par le chercheur et par le professeur lui-même. Nous sommes alors proches d'un entretien d'auto analyse. Revenir ainsi sans cesse aux images (par des accélérés, des ralentis, des retours en arrière,...) apporte à la fois la familiarité nécessaire à la compréhension de l'histoire de la séance, mais, dans un mouvement inverse, opère également une forme d'estrangement (Ginzburg, 2001), c'est-à-dire une prise de distance par rapport à ce familier.

Notre analyse, quant à elle, s'élabore selon trois temps et considère successivement analyse structurelle, analyse de la dynamique du système JA/JE et analyse de l'épistémologie pratique du professeur. L'analyse structurelle se déploie dans le langage naturel de l'action. Elle engage trois étapes : la première (a) concerne la présentation du contexte de l'épisode analysé ; la seconde (b) propose une analyse didactique a priori de la notion étudiée dans le cadre de la situation proposée par le professeur. Il s'agit d'identifier les occasions potentielles d'apprendre offertes aux élèves, mais aussi les obstacles possibles. La troisième (c), c'est l'intrigue didactique qui représente la mise en récit de l'action conjointe du point de vue du savoir. La vision sous forme d'intrigue "consiste bien à mettre ensemble des faits dont la perception courante ne peut opérer le rapprochement » (Sensevy, 2011). L'ensemble de cette analyse structurelle se donne pour mission de «clarifier une pratique donnée par la mise en visibilité et en contiguité de formes autrement " cachées » de cette pratique » (Sensevy, ibid.).

L'analyse des pratiques effectives utilise les outils de la TACD. Elle relève d'une analyse dynamique du système JA-JE et - a contrario de l'analyse structurelle - s'établit dans le langage théorique de l'action conjointe. Elle met au jour l'évolution des différents Jeux d'Apprentissage référés au Jeu Épistémique visé et permet ainsi de mieux appréhender le processus d'élaboration des connaissances des élèves. Les traces de cette évolution seront reconstruites à partir des verbatim, analysés du point de vue des interactions verbales.

L'analyse structurelle et celle de la dynamique JA/JE permettent au chercheur d'apprécier l'écart entre les apprentissages attendus et réalisés. Rendre compte de la manière dont se sont produits ces écarts, est une condition, certes nécessaire, mais non suffisante à la compréhension de l'action. Élucider certains éléments de l'épistémologie pratique du professeur peut nous permettre de saisir certaines des déterminations de l'action conjointe, jusqu'alors inaccessibles. Le croisement des données recueillies lors des entretiens ante et post (les éléments d'épistémologie pratique qui orientent l'action) avec les analyses des pratiques effectives (les éléments d'épistémologie pratique sélectionnés ou renforcés par la situation telle qu'elle se joue), va permettre au chercheur de réinformer l'analyse didactique (la dynamique JA/JE). Ce réenrichissement de l'analyse didactique autorise alors la production de nouvelles inférences sur les rapports que le professeur adopte vis-à-vis du savoir enseigné, mais aussi, dans notre cas, vis-à-vis de la difficulté d'apprentissage et plus généralement visà-vis du métier. C'est ainsi que nous tentons de construire un pont entre sens pratique du professeur et conceptualisations du chercheur. 


\section{Études de cas}

Dans cette partie, nous allons présenter successivement nos deux études de cas, «les rectangles » et "les aiguilles » selon les trois temps précédemment cités : l'analyse structurelle, l'analyse de la dynamique du système JA/JE enfin l'analyse de l'épistémologie pratique du professeur.

\section{Étude de cas $\mathrm{n}^{\circ} 1:$ «les rectangles »}

\section{Analyse structurelle}

\section{Présentation du contexte}

Figure 2 : Page du manuel présentant la situation des rectangles

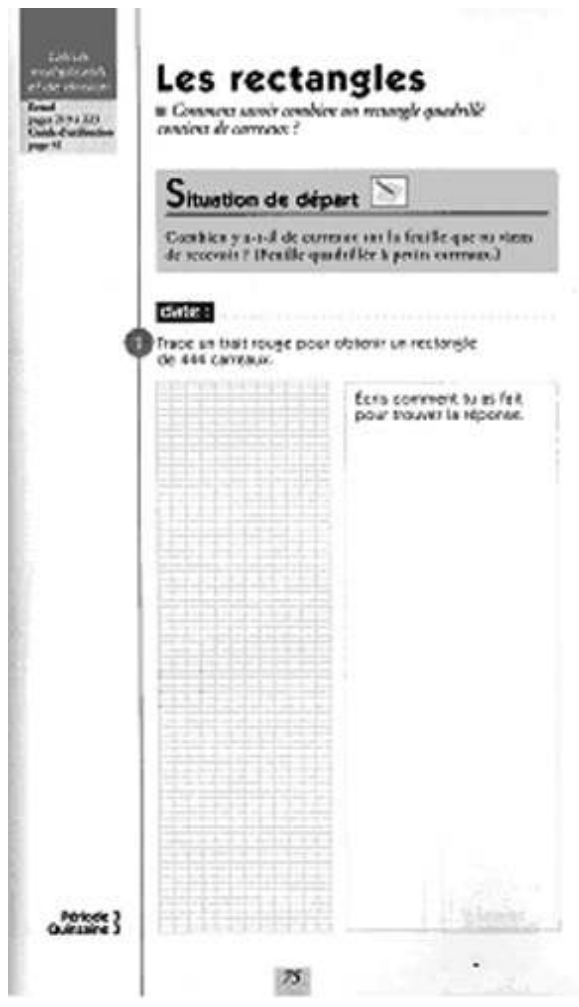

Les objectifs de cette situation « Les rectangles » sont ainsi rédigés dans le manuel :

- Utiliser des écritures multiplicatives dans des situations de produits de mesures ;

- Savoir utiliser la multiplication pour trouver le nombre d'éléments d'une configuration rectangulaire ;

- Savoir résoudre des problèmes de division liés à cette configuration (sans connaître la technique opératoire);

- Élaborer des procédures personnelles de résolution de problèmes de division et les améliorer. 
L'épisode d'aide étudié dure 26 minutes et 31 secondes. Le professeur (P1) a organisé un temps d'étude spécifique pour Martine et Marine, un binôme qu'il qualifie de "peu performant » en mathématiques. Pendant ce temps, les élèves de cette classe de CE2 travaillent à la résolution d'exercices de français proposés dans leur plan de travail hebdomadaire. C'est cette organisation particulière de la classe ${ }^{9}$ qui permet au professeur de se libérer régulièrement et quotidiennement pour aider les élèves repérés " en difficulté ». Martine et Marine ont à résoudre, avec l'aide de P1, une forme adaptée de la situation-problème «les rectangles » (Ermel CE2, p. 219 à 223). Les deux élèves disposent de la photocopie du manuel présentée ci dessous.

La consigne de la situation originale s'exprime ainsi : "trace un trait rouge pour obtenir un rectangle de 444 carreaux». Cette situation initiale est simplifiée pour Marine et Martine. Le professeur a joué sur la variable numérique et a remplacé le nombre $444(37 \times 12)$ par celui de $48(4 \times 12)$. P1 propose donc la consigne suivante: « Trace un trait rouge pour obtenir un rectangle de 48 carreaux.»

\section{Analyse a priori}

Dans cette situation «Les rectangles ", il s'agit, par la comparaison des résultats et des procédures, de mettre en évidence que compter le nombre de carreaux des deux dimensions du rectangle (L et l) et effectuer leur produit est une procédure performante. Nous avons dit précédemment que P1 a opéré une simplification des données numériques passant d'un rectangle de 444 carreaux à 48 carreaux. Quels effets cette modification pourrait-elle avoir sur les procédures possibles des élèves?

Le tableau suivant présente, pour chacune des deux situations - celle proposée par le manuel et celle proposée aux deux élèves en difficulté - les différentes procédures de résolution et leur efficacité.

Tableau 1 : Les procédures possibles des élèves dans les 2 situations

\begin{tabular}{|c|c|c|}
\hline $\begin{array}{l}\text { Procédures de résolution } \\
\text { possibles }\end{array}$ & $\begin{array}{l}\text { Situation « originale " }(444 \\
\text { carreaux })\end{array}$ & $\begin{array}{l}\text { Situation "transformée " } \\
(48 \text { carreaux) }\end{array}$ \\
\hline Compter les carreaux de 1 en 1 . & $\begin{array}{l}\text { Inefficace étant donné le } \\
\text { nombre de carreaux. }\end{array}$ & $\begin{array}{l}\text { Envisageable puisque le } \\
\text { nombre de carreaux est } \\
\text { petit. }\end{array}$ \\
\hline $\begin{array}{l}\text { Remplissage de la feuille quadrillée } \\
\text { à l'aide de bandes de } 10 \text { carreaux. }\end{array}$ & $\begin{array}{l}\text { Manière efficace de dénombrer } \\
\text { une collection importante, mais } \\
\text { la tâche se révèle encore lourde. }\end{array}$ & $\begin{array}{l}\text { Le nombre peu important } \\
\text { de carreaux ne le nécessite } \\
\text { pas. }\end{array}$ \\
\hline $\begin{array}{l}\text { Remplissage de la feuille quadrillée } \\
\text { à l'aide de carrés de } 100 \text { carreaux, } \\
\text { puis comptage ou groupements } \\
\text { divers sur les carreaux qui restent. }\end{array}$ & $\begin{array}{l}\text { Manière efficace de dénombrer } \\
\text { une collection importante. }\end{array}$ & $\begin{array}{lll}\text { Ce remplissage } & \text { ne } \\
\text { correspond pas à un } \\
\text { nombre inférieur à 100. }\end{array}$ \\
\hline $\begin{array}{l}\text { Comptage des carreaux sur une } \\
\text { dimension du rectangle et addition } \\
\text { réitérée. }\end{array}$ & $\begin{array}{l}\text { Difficile car il faut additionner } \\
37 \text { douze fois. }\end{array}$ & $\begin{array}{l}\text { Forme simple de calcul: } \\
12+12+12+12 \text { envisageable. }\end{array}$ \\
\hline Écriture multiplicative. & $\begin{array}{l}\text { Nécessité d'une procédure } \\
\text { multiplicative }(12 \times 37) \text {. }\end{array}$ & $\begin{array}{l}\text { Pas de nécessité d'une } \\
\text { procédure multiplicative } \\
(12 \times 4) \text {. }\end{array}$ \\
\hline
\end{tabular}



affaiblit l'objectif principal de cette situation. En effet, pour 48 carreaux, il n'y a plus de nécessité d'utiliser une procédure multiplicative. Concentrons-nous maintenant sur la tâche que les deux élèves sont censés réaliser, c'est-à-dire tracer un trait rouge pour obtenir un rectangle. Le quadrillage comporte 12 carreaux de large et pour obtenir un rectangle de 48 carreaux, il suffit de sélectionner les 4 premières lignes de ce quadrillage. L'élève trace alors un unique segment, les trois autres côtés étant matérialisés par les bordures du quadrillage. C'est cette solution qui est attendue par le manuel Ermel ${ }^{10}$. En revanche, si l'élève ne comprend pas que le terme «trait » implique le traçage d'un unique segment, mais le traduit comme celui permettant de tracer un périmètre qui correspond à une aire de 48 carreaux, alors le rectangle peut prendre diverses positions dans le quadrillage. Dans ce cas, le fait que le quadrillage ait 12 carreaux de largeur perd de son caractère de nécessité. L'élève peut alors obtenir des produits tels $24 \times 2$ ou $4 \times 12$ et l'enjeu didactique de la situation est déplacé.

\section{Intrigue didactique}

P1 distribue la photocopie sans faire de commentaires et laisse Marine et Martine découvrir seules la situation "Les rectangles", lire la consigne et démarrer la résolution, pendant qu'il aide une autre élève. Huit minutes plus tard, P1 retourne auprès des deux élèves. Martine n'a rien écrit sur sa feuille, Marine a tracé un trait rouge sur un côté du quadrillage (la longueur de ce segment est de 48 carreaux). Elle n'a pas pris en compte les deux conditions simultanées, d'une part, tracer un seul trait rouge et, de l'autre, obtenir un rectangle. P1 demande alors aux deux élèves de lire successivement à haute voix la consigne "tracer un trait rouge pour obtenir un rectangle de 48 carreaux ».

À partir de ce moment, le professeur va faire évoluer la tâche initiale proposée aux deux élèves. Il redéfinit ainsi successivement ses attentes :

quand il s'aperçoit que la consigne n'est assimilée qu'en partie, P1 ne tente pas de reformulation, mais demande à Marine et Martine de tracer un trait rouge pour obtenir un rectangle de 6 carreaux, puis de tracer un rectangle et de repasser les contours en rouge.

P1 demande ensuite aux élèves, une fois cette dernière tâche réalisée, de partager le rectangle en 6 carreaux. Marine et Martine échouent. P1 reformule la question et leur signifie qu'il attend un partage du rectangle en 6 morceaux égaux. P1 s'éloigne pendant quatre minutes pour aider deux autres élèves.

Quand P1 retourne auprès de Martine et Marine, il constate la difficulté de faire 6 parties égales et modifie une nouvelle fois la consigne. Il leur demande alors de colorier 6 carreaux sur le quadrillage de la fiche pour faire un rectangle, de le découper et de le coller. L'épisode s'achève ainsi.

\section{Analyse didactique des pratiques effectives : étude dynamique du système JA/JE}

L'analyse qui suit décrit les différentes étapes qui conduisent à des réaménagements successifs des milieux de l'étude. La représentation de cette évolution est donnée à travers un système sémiotique (Marlot, 2008) ${ }^{11}$ qui positionne selon le gradient " Jeux d'Apprentissage génériques - Jeux d'Apprentissage spécifiques », les différents Jeux

Éducation et didactique, 5-3 | 2011 
d'Apprentissage de chaque épisode étudié, identifiés par le chercheur. Il y a ainsi une infinité de positionnements possibles des JA entre les 2 pôles du gradient, les Jeux d'Apprentissage pouvant être plus ou moins génériques ou plus ou moins spécifiques, relativement au Jeu Épistémique visé par la situation. Le système sémiotique donne alors à voir, pour chacun des JA, deux variables : (1) la densité épistémique du savoir mobilisé par le Jeu et (2) l'ordre de son « apparition » au cours de l'action conjointe (de JA1 à JA $n+1)$. Au-delà d'une simple apparition chronologique des JA, il se produit des substitutions de JA spécifiques par des Jeux plus génériques. Ce phénomène didactique a été appréhendé sous le terme de glissement de Jeux d'Apprentissage (Marlot, 2008).

L'analyse qui suit n'est autre que la narration réitérée de l'intrigue didactique présentée plus haut (c. $\mathrm{f}$ (c) analyse structurelle), mais cette fois-ci dans le langage théorique de l'action conjointe. Ce changement de registre nous permet de passer des faits exposés aux phénomènes didactiques identifiés. Ce faisant, nous nous rapprochons ainsi de notre intention de départ qui est de comprendre comment «vivent » (et se transforment) les objets du milieu en lien avec les règles d'usage qui les animent.

Figure 3 : Évolution des Jeux d'Apprentissage effectifs - cas du professeur P1 « les rectangles»
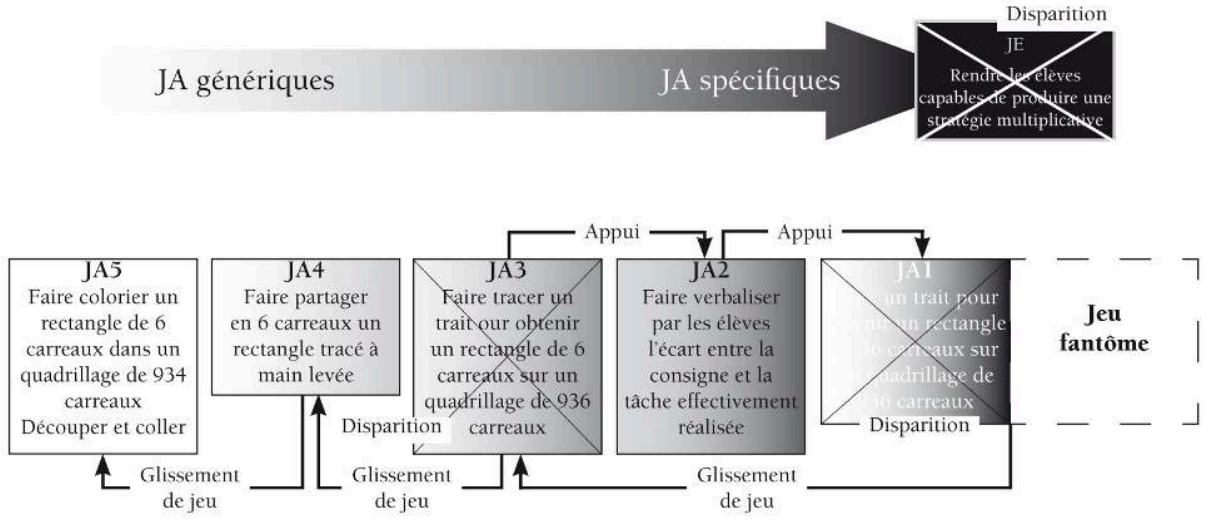

Étape 1. Le premier Jeu d'Apprentissage, nous l'avons nommé JA1 : «faire tracer un trait pour obtenir un rectangle de 48 carreaux sur le quadrillage de 936 carreaux ». Cette tâche est échouée par les deux élèves qui ne produisent pas la réponse attendue (Marine trace un simple trait qui couvre 48 carreaux).

Étape 2. P1 demande alors aux deux élèves d'évaluer elles-mêmes l'écart entre ce qui est attendu par la consigne et ce qu'elles ont effectivement réalisé (Qu'est-ce que vous avez à faire ? (tdp $\left.{ }^{12} 96\right)$; Alors, qu'est-ce que tu as fait sur ta feuille? (tdp 102) ; Donc, tu as terminé ton exercice en fait? (tdp 106); Pourquoi tu n'as pas fini ? (tdp108)). C'est $\mathrm{JA} 2^{13}:$ « faire verbaliser par les élèves l'écart entre la consigne et la tâche effectivement réalisée ».

Étape 3. Nous constatons alors la disparition de JA1 au profit de JA3 : «faire tracer un trait pour obtenir un rectangle de 6 carreaux sur un quadrillage de 936 carreaux ». Le professeur provoque donc un changement de milieu, mais qui induit (sans doute à son insu) un changement de contrat: en effet l'action de P1 génère une sortie de la situation de recherche de stratégie multiplicative. Nous assistons donc à un premier glissement de Jeu d'Apprentissage avec une transformation de l'enjeu didactique initial. 
Tableau 2 : Évolution du statut de l'objet « quadrillage » dans le cas des « rectangles »

\begin{tabular}{|l|l|}
\hline & Verbatim \\
\hline \multirow{3}{*}{$\begin{array}{l}\text { Statut 1 } \\
\text { Rectangle dans le quadrillage }\end{array}$} & $\begin{array}{l}\text { « Vous devez tracer un rectangle, tracer un trait rouge pour } \\
\text { obtenir un rectangle de 48 carreaux » (tdp 113). }\end{array}$ \\
\cline { 2 - 3 } & $\begin{array}{l}\text { «aites-moi, euh, un rectangle de 6 carreaux par exemple. Tracez- } \\
\text { moi un trait rouge pour obtenir un rectangle de 6 carreaux » (tdp } \\
113) .\end{array}$ \\
\hline
\end{tabular}

Étape 4. Le professeur engage ensuite un nouveau Jeu, JA4 «faire partager en 6 carreaux un rectangle tracé à main levée ». JA4 est un « déguisement » de JA3 : dans les deux cas il y a production d'un rectangle de 6 carreaux, mais pour JA4 les élèves ne sont plus placés dans le contexte du quadrillage. C'est pourquoi JA4 est moins dense épistémiquement que JA3. Le professeur permet ainsi aux deux élèves de ne pas perdre la face, le lien didactique est apparemment maintenu. Les élèves produisent une trace écrite. Nous assistons à un second glissement de Jeu.

Étape 5. P1 convoque alors un autre Jeu d'Apprentissage très générique, JA5 : «faire colorier un rectangle de 6 carreaux dans le quadrillage de 936 carreaux, puis le découper et le coller sur le cahier». P1 convoque alors à nouveau l'objet «quadrillage ». Ce que P1 demande aux deux élèves correspond à des connaissances antérieures, normalement stabilisées en CE2. Apparaît alors un nouveau milieu (l'objet quadrillage a changé de statut, il devient support de la production de rectangles) et un nouveau contrat (il s'agit maintenant d'isoler un rectangle). Nous assistons à un troisième glissement de Jeu.

Cette forme sémiotique met au jour que le premier Jeu d'Apprentissage JA1 devient un "Jeu fantôme ", c'est-à-dire vidé de sa substance d'apprentissage. Il y a in fine une manifestation du fonctionnement du contrat didactique qui s'exprime sous la forme d'un effet de contrat, l'effet Jourdain (Brousseau, 1998) : le professeur fait comme si les élèves avaient produit la réponse initialement attendue. Cet effet de contrat se traduit par des glissements graduels de Jeux, d'un Jeu spécifique (JA1) vers un Jeu générique (JA5). Nous constatons que les Jeux d'Apprentissage effectifs s'éloignent progressivement - pour se dissocier - du Jeu Épistémique qui était de rendre les élèves capables de produire une stratégie multiplicative. Il y a donc évanouissement du savoir visé.

Du point de vue des règles des Jeux d'Apprentissage, l'enseignante ne rappelle à aucun moment la règle définitoire, c'est-à-dire produire un seul trait rouge sur le quadrillage. Avec la réduction de la valeur numérique (passage de 444 carreaux à 48 carreaux), la règle stratégique "trouver un moyen efficace pour compter les carreaux de manière exacte » ne tient pas (puisqu'il n'y a pas nécessité d'utiliser la multiplication). Avec 48 carreaux le comptage de 1 en 1 est en effet suffisant. Sans production de règle stratégique par les élèves, le Jeu Épistémique ne peut être joué. Ainsi le quadrillage va alors convoquer chez les deux élèves d'autres actions, en référence à des usages qu'ils ont déjà expérimentés ${ }^{14}$.

Dans le tableau ci-dessous, nous mettons au jour le fait que P1 va jouer sur les aspects matériels. L'objet quadrillage va alors prendre plusieurs statuts successifs. 


\begin{tabular}{|l|l|}
\hline $\begin{array}{l}\text { Statut } 2 \\
\text { Rectangle hors quadrillage }\end{array}$ & $\begin{array}{l}\text { «Dessine-moi un rectangle au crayon à papier, c'est tout, sans } \\
\text { règle, à la main levée » (tdp 115). }\end{array}$ \\
\hline $\begin{array}{l}\text { Statut } 3 \\
\text { Pavage }\end{array}$ & «Regarde, fais-moi 6 carreaux à l'intérieur » (tdp 127). \\
\hline $\begin{array}{l}\text { Statut } 4 \\
\text { Pavage pour constituer un } \\
\text { rectangle dans le quadrillage }\end{array}$ & «Regarde, fais-moi 6 carreaux à l'intérieur » (tdp 127). \\
\hline
\end{tabular}

P1 fait évoluer l'objet initial « quadrillage » d'un statut mathématique (avec l'ambition première de la nécessité de l'utilisation de la stratégie multiplicative) à un statut matériel (une forme rectangulaire à découper et colorier). Nous constatons un "éparpillement des tâches". Tout se passe comme si on assistait à une sorte de « dilatation du temps " (à l'inverse du cas des aiguilles, où comme nous le verrons plus tard, c'est plutôt de "contraction du temps" dont on pourrait parler). La posture d'aide de P1 s'apparente à de la sollicitude ${ }^{15}$ que Renaut (2002) nomme le soutien moral (Toullec-Théry, à paraître). Les modifications de milieu provoquées par P1 semblent affaiblir les enjeux de savoir. Dans les échanges entre le professeur et les élèves, la dimension conversationnelle semble prendre le pas sur la dimension épistémique. L'idée de P1, qu'aider, c'est simplifier la situation amène dans les faits à une dilution de l'objet d'apprentissage.

\section{Analyse de l'épistémologie pratique du professeur P1}

\section{Éléments d'épistémologie pratique qui orientent l'action}

Le discours du professeur sur son action met au jour ses intentions, ses croyances, les contraintes qu'il s'impose. Nous avons sélectionné dans les entretiens, les énoncés qui semblaient être de bons candidats à l'explicitation du rapport que le professeur pouvait entretenir, d'une part à la difficulté scolaire et d'autre part au savoir enseigné et à l'apprentissage. D'un point de vue thématique, nous pouvons dire que chez P1, les énoncés relatifs à la première catégorie (le rapport à la difficulté scolaire) sont bien plus nombreux que ceux relatif à la deuxième catégorie (le rapport au savoir et à l'apprentissage). Le tableau qui suit propose dans la première colonne, les énoncés les plus emblématiques de ces 2 catégories et dans la seconde colonne - pour chacun de ces énoncés - des éléments de l'épistémologie pratique de P1; étant bien entendu que ces éléments sont un construit du chercheur.

Tableau 3 : Éléments d'épistémologie pratique du professeur P1

\begin{tabular}{|c|c|}
\hline Verbatim & Éléments de l'épistémologie pratique de P1 \\
\hline \multicolumn{2}{|l|}{ Rapport de P1 à la difficulté scolaire } \\
\hline $\begin{array}{l}\text { Donc l'idée, avec les plans de travail, c'est de garder } \\
\text { mon temps, ma disponibilité et ma compétence entre } \\
\text { guillemets d'enseignante pour les enfants qui, sur un } \\
\text { sujet, posent une difficulté. }\end{array}$ & $\begin{array}{l}\text { - La difficulté scolaire fait signe. } \\
\text { - L'aide ordinaire passe par la présence de } \\
\text { l'enseignant. } \\
\text { - L'organisation de la classe selon les } \\
\text { techniques de la classe coopérative favorise la } \\
\text { prise en charge des élèves en difficulté. }\end{array}$ \\
\hline
\end{tabular}




\begin{tabular}{|c|c|}
\hline $\begin{array}{l}\text { Comme ils ne font pas tous la même chose au même } \\
\text { moment, je peux répondre à leur question et non à un } \\
\text { ensemble. } \\
\text {... Avec ces élèves les petits trucs ça ne marche pas, elles } \\
\text { ne réfléchissent pas de la même manière que les autres. } \\
\text { [...] Je ne peux m'appuyer sur rien, je ne sais pas ce qui } \\
\text { leur manque, elles ne disent rien [...]. }\end{array}$ & $\begin{array}{l}\text { - Il faut que l'élève mette en mots sa difficulté } \\
\text { et pour cela il y a nécessité à construire les } \\
\text { conditions de cette expression. } \\
\text { - C'est seulement si l'élève exprime sa } \\
\text { difficulté que l'on peut repérer l'obstacle et } \\
\text { l'aider. }\end{array}$ \\
\hline $\begin{array}{l}\text { Ma place, j'essaie toujours de la définir, dans un premier } \\
\text { temps, comme de la reformulation, c'est-à-dire, je vérifie } \\
\text { si c'est un problème de vocabulaire ou un problème de } \\
\text { compréhension. }\end{array}$ & \multirow{2}{*}{$\begin{array}{l}\text { L'aide se structure selon le format suivant: } \\
\text { 1. Identification de la nature et du niveau de } \\
\text { difficulté en terme de "compréhension ou } \\
\text { vocabulaire » (diagnostic); } \\
\text { 2. Proposition de situations-problème comme } \\
\text { mode de remédiation. }\end{array}$} \\
\hline $\begin{array}{l}\text { Le mot clé, c'est le problème, qu'ils [les élèves] soient } \\
\text { confrontés tout le temps à des problèmes. }\end{array}$ & \\
\hline \multicolumn{2}{|l|}{ Rapport de P1 au savoir enseigné } \\
\hline $\begin{array}{l}\text { - Un trait rouge, c'est pas compliqué. } \\
\text { - C'est une situation qui est simple à mettre en œuvre. }\end{array}$ & $\begin{array}{l}\text { Une opération mathématique complexe peut } \\
\text { se traduire par une tâche simple. }\end{array}$ \\
\hline $\begin{array}{l}\text { J'essaie de voir ce qui leur manque, les pré-requis } \\
\text { qu'elles n'ont pas. }\end{array}$ & $\begin{array}{l}\text { L'apprentissage est linéaire, il s'agit pour } \\
\text { aider l'élève de combler les manques. }\end{array}$ \\
\hline
\end{tabular}

40 Ces éléments d'épistémologie pratique du professeur P1 nous autorisent à afficher quelques traits saillants, et de mieux comprendre ainsi certaines déterminations qui pèsent sur l'action de cet enseignant lorsqu'il aide les élèves en difficulté. Ces éléments d'épistémologie pratique s'agrègent pour donner lieu à une sorte de format :

- créer les conditions d'une aide sur le temps de la classe grâce à une organisation basée sur l'usage des « plans de travail » et qui conduit le professeur à se rendre disponible auprès de groupe homogène faible.

- diagnostiquer la difficulté selon deux indicateurs : problème de vocabulaire vs problème de compréhension.

- aider l'élève à mettre en mot sa difficulté afin de repérer les obstacles à l'apprentissage

- remédier à la difficulté en proposant des situations-problème.

- Ce format joue le rôle d'une configuration qui organise la situation d'aide et définit les responsabilités qui incombent au professeur.

\section{Ré-information de l'analyse didactique}

41 Ce «format de l'aide» oriente dans un premier temps, l'action du professeur : il propose une situation-problème pour un binôme d'élèves en difficulté. Il s'attend à ce que les élèves naturellement - dans le cadre du Jeu d'Apprentissage JA2 « Faire verbaliser par les élèves l'écart entre la consigne et la tâche effectivement réalisée » - expriment ce qu'elles comprennent de la situation et prennent ainsi conscience de leurs difficultés.

Or, dans l'épisode analysé, les 2 élèves ne parlent pas. Le professeur se trouve alors démuni (en référence à son format de l'aide) pour identifier ce qui fait difficulté chez ces élèves, ce qu'elles ne comprennent pas. Cet événement va réactiver l'élément de l'épistémologie pratique selon lequel, pour P1, «aider c'est permettre à l'élève de mettre en mots sa difficulté ». Pour permettre aux élèves de "s'exprimer ", le professeur 
engage les élèves sur le versant des difficultés liées au vocabulaire (mathématique) en focalisant leur attention sur "le rectangle ", notion pourtant maîtrisée par ces élèves de CE2. Pour ce faire, il confronte les élèves à des tâches multiples et de plus en plus «simples». Ainsi, dans l'action, se cristallisent ou se remobilisent de nouveaux éléments d'épistémologie pratique. Dans ce cas tout se passe comme si le professeur mobilisait l'idée suivante : plus les tâches sont simples, mieux les élèves exprimeront leurs difficultés ou la compréhension de ce qui est attendu.

Nous comprenons alors mieux pourquoi les actions d'aide de P1 relèvent d'un modèle générique qui ne prend pas vraiment en compte la spécificité de la situation avec ses enjeux de savoir afférents. Le professeur ne travaille pas avec les obstacles liés à la situation de savoir (puisqu'il déplace immédiatement l'enjeu didactique initial), mais plutôt avec ce qu'il identifie comme des "manques » chez les élèves (l'identification et le tracé d'un rectangle).

Tout se passe comme si, pour ce professeur, très attentif à la difficulté scolaire, l'aide consistait à permettre aux élèves de mettre en mots leur(s) difficulté(s). Ainsi, il semblerait que chez ce professeur la mise en scène de l'attention à la difficulté prenne le pas sur la mise en scène du savoir et le souci de «faire réussir » prenne le pas sur le souci de « faire apprendre».

\section{Étude de cas $\mathrm{n}^{\circ} 2$ : « les aiguilles »}

\section{Analyse structurelle}

\section{Présentation du contexte}

L'épisode d'aide étudié dure 10 minutes. Le professeur (P2) a organisé, immédiatement après la séance en grand groupe, un temps d'étude spécifique pour un binôme (Morgane et Julie) qui a échoué les exercices donnés lors de la séance. Ces deux élèves s'avèrent être, selon l'enseignant, des élèves globalement "peu performantes " en mathématiques. Pendant ce temps, les autres élèves de cette classe de CE2 travaillent en groupes de 4 à un jeu sur les heures. Comme P1, ce professeur met en place une organisation particulière de la classe qui lui permet de se libérer régulièrement pour aider les élèves repérés « en difficulté ».

P2 est installé à une grande table entre Morgane, assise à sa droite, et Julie, assise à sa gauche. Il tient entre ses mains une pendule analogique classique avec des chiffres de 1 à 12. Les aiguilles sont désolidarisées et portent des couleurs différentes (la grande aiguille est rouge, la petite aiguille verte). Il n'y a pas, sur la pendule, d'aménagement supplémentaire, comme l'affichage des minutes par exemple.

P2 reprend, dans le temps court de l'épisode d'aide, les objectifs identiques à ceux qu'il poursuivait lors de la séance en grand groupe sachant que les trois premiers objectifs de sa séance recouvraient des révisions (et n'impliquaient, pour P2, qu'un entraînement).

- objectif 1 : Lire et/ou afficher des heures sur des horloges analogiques.

- Objectif 2 : Positionner la grande aiguille pour indiquer quart d'heure et demi-heure.

- objectif 3 : Calculer des durées. 


\section{ciblé pour cette séance :}

- Objectif 4 : Transformer l'heure ante meridiem en heure post meridiem.

\section{Analyse a priori}

Dans cette situation d'affichage et de lecture d'heures, il s'agit de mettre en évidence que pour une même heure affichée, il y a deux expressions possibles, l'une ante meridiem, l'autre post meridiem. Pour répondre à cette attente, les élèves doivent disposer de connaissances préalables et de procédures de résolution.

Le nombre de connaissances à maîtriser préalablement pour espérer atteindre les 4 objectifs d'apprentissage ciblés est très important. De plus, la lecture de l'heure produit une rupture avec la numération décimale. Pour la majorité des grandeurs, les multiples et sous multiples se suivent en effet et changent dans un rapport de 1 à 10 ou $1 / 10$, or ce n'est pas le cas pour les unités de temps.

Tableau 4 : Identification des connaissances requises

\begin{tabular}{|c|c|}
\hline Objectifs d'apprentissage & Connaissances nécessaires \\
\hline \multirow[t]{4}{*}{$\begin{array}{l}\text { Objectif 1- Lire et/ou afficher des heures } \\
\text { sur des horloges analogiques. } \\
\text { et objectif 2- Positionner la grande } \\
\text { aiguille pour indiquer quart d'heure et } \\
\text { demi-heure.. }\end{array}$} & La grande aiguille indique les minutes. \\
\hline & La petite aiguille indique les heures. \\
\hline & $\begin{array}{l}\text { Lorsque la grande aiguille se situe sur } 12 \text {, c'est l'heure } \\
\text { «pile», lorsqu'elle se situe sur le } 6 \text {, c'est la demi-heure; } \\
\text { sur le } 3 \text { c'est le quart d'heure. }\end{array}$ \\
\hline & $\begin{array}{l}\text { Lorsque la petite aiguille se situe entre deux nombres } \\
\text { indiquant les heures, c'est la demi. }\end{array}$ \\
\hline \multirow[t]{2}{*}{$\begin{array}{l}\text { Objectifs } 1 \text { et } 2 \text { mais aussi } \\
\text { Objectif } 3 \text { - Calculer des durées. }\end{array}$} & $\begin{array}{l}\text { Pour une graduation, la petite aiguille se déplace d'une } \\
\text { heure (ce qui correspond à }+1 \text { ). }\end{array}$ \\
\hline & $\begin{array}{l}\text { Pour une graduation, la grande aiguille se déplace de } 5 \\
\text { minutes (ce qui correspond à }+5 \text { ). }\end{array}$ \\
\hline \multirow[t]{2}{*}{$\begin{array}{l}\text { Objectifs 1,2,3 mais aussi } \\
\text { Objectif } 4 \text { - Transformer l'heure ante } \\
\text { meridiem en heure post meridiem }\end{array}$} & $\begin{array}{l}\text { Les heures indiquées sur la pendule peuvent être lues } \\
\text { soit en heures ante meridiem soit en heure post meridiem. }\end{array}$ \\
\hline & $\begin{array}{l}\text { Quand la grande aiguille fait un tour, elle avance d'une } \\
\text { heure ( } 60 \text { minutes }=1 \text { heure). }\end{array}$ \\
\hline Objectif 4 & $\begin{array}{l}\text { Objectif } 4 \mathrm{X}+12(\text { avec } \mathrm{x}<12) \text { permet de passer de l'heure } \\
\text { ante meridiem à l'heure post meridiem. }\end{array}$ \\
\hline
\end{tabular}

51 Le milieu proposé lors de cette séance d'aide est le même que celui proposé à l'ensemble de la classe lors de la séance collective: la pendule analogique avec des aiguilles désolidarisées. Le professeur n'a pas réaménagé le milieu. La charge cognitive 
des deux élèves reste donc inchangée. Ainsi, le fait que les aiguilles soient désolidarisées complexifie la situation. Il est nécessaire de déplacer l'une puis l'autre, avec les imprécisions que ce déplacement occasionne. P2 ne modifie pas non plus le cadran. Aucun nombre affiché n'existe pour les minutes, ce qui nécessite pour l'élève de savoir déjà associer à chaque position de la grande aiguille un nombre de minutes. Toutefois, si ces positionnements ne sont pas mémorisés, il doit utiliser une stratégie plus coûteuse qui est de systématiquement recalculer le nombre de minutes en comptant de 5 en 5 à partir de l'heure pleine. Il apparait donc que le milieu offert introduit des obstacles didactiques qui peuvent rendre ce milieu non opératoire.

\section{Intrigue didactique}

52 Au début de l'épisode, les aiguilles de la pendule marquent 1h00, la tâche prescrite par le professeur consiste à faire afficher 15h30. P2 demande à Morgane, dans une première étape, de déplacer les aiguilles de la pendule pour passer de 1 h00 à $1 \mathrm{~h} 30$ et de passer ainsi de l'heure "pile ", indiquée initialement sur la pendule, à la demi-heure dont ils auront besoin pour matérialiser l'heure finale attendue, à savoir $15 \mathrm{~h} 30$. Ce passage de 1 h00 à 1 h30 n'augure a priori pas, selon $\mathrm{P} 2$, de difficulté dans la mesure où il coïncide avec un des objectifs d'entraînement de la séance en grand groupe. Or, P2 découvre que cet objectif (afficher l'heure " pile » et la demi-heure) n'est pas maîtrisé. Sur ce temps court de l'aide, il va pourtant continuer à tenir les quatre objectifs. Malgré les difficultés et les savoirs "manquants", il poursuivra sur le passage ante meridiem/post meridiem, Pour introduire cette notion, il va d'abord donner un exemple « $15 \mathrm{~h} 30$ c'est la même chose que $3 \mathrm{~h} 30$ » puis, dans un temps très court, il va mêler dans son discours la dénomination heure am et heure $p m$ pour produire l'effet d'une assimilation de l'une à l'autre ( $15 \mathrm{~h} 30$ c'est pareil que $3 \mathrm{~h} 30$; il faut passer de $1 \mathrm{~h} 30$ à $2 \mathrm{~h} ; 2 \mathrm{~h}$ c'est pareil que $14 \mathrm{~h}$; essayez de passer de $14 \mathrm{~h}$ à $15 \mathrm{~h}$ et $3 \mathrm{~h}$ c'est pareil que 15h). Pourtant P2 sera contraint d'abandonner la tâche en cours devant l'impossibilité des élèves d'afficher 15h30. Il leur demandera alors de donner la couleur des aiguilles de la pendule.

\section{Analyse didactique des pratiques effectives : Étude de la dynamique du système JA/JE}

Nous rappelons que le projet d'aide de P2 est de permettre aux 2 élèves en difficulté, repérées pour avoir échoué la transformation de l'heure ante meridiem en heure post meridiem, de revenir sur l'apprentissage de cette notion. 
Figure 4 : Évolution des Jeux d'Apprentissage effectifs - cas du professeur P2 « les aiguilles »

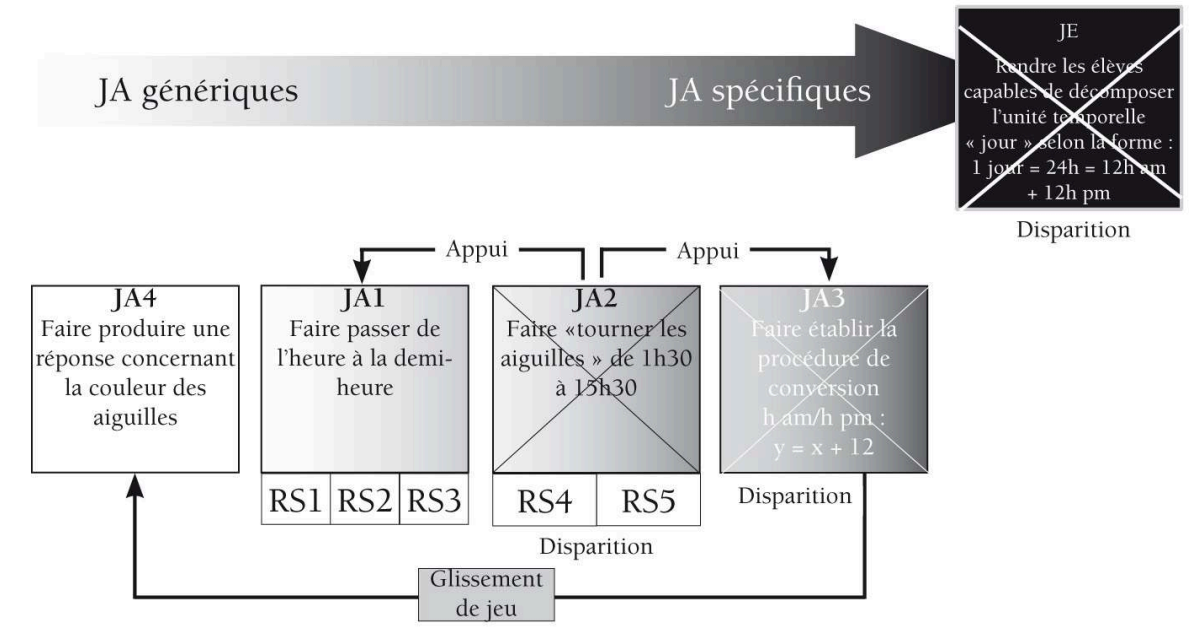

Étape 1. Le professeur commence par une révision du travail réalisé en collectif. Il s'agit d'afficher des heures sur l'horloge. Le Jeu d'Apprentissage mobilisé, JA1 est le suivant : « faire avancer la grande aiguille de 30 minutes en avançant de 5 en 5 ». Ce Jeu d'Apprentissage est peu spécifique, car dans le contexte de la leçon, il a valeur de révision. Toutefois, pour ces élèves en difficulté, il s'avère correspondre à un véritable apprentissage.

La règle définitoire est portée par l'objet lui-même, l'horloge et ses 2 aiguilles de couleur différente que les élèves ont déjà vu manipuler par le professeur. La règle définitoire concerne l'avancée des aiguilles sur le cadran: l'avancée d'une aiguille s'effectue selon un double codage. L'horloge étant découpée en 60 graduations, la grande aiguille avance d'une minute quand elle effectue un trajet d' $1 / 60$, alors que la petite aiguille avance d'une heure quand elle parcourt $5 / 60$. Or, les élèves ne connaissant pas ces codages ne peuvent entrer dans le Jeu d'Apprentissage JA1. En conséquence, ils ne sont pas en mesure de produire les règles stratégiques qui leur permettraient de "gagner au Jeu ", c'est-à-dire de positionner correctement les 2 aiguilles sur le cadran pour indiquer l'heure demandée (13h30). C'est donc P2 qui va prendre en charge la diffusion de ces règles aux deux élèves. Soit, la règle stratégique 1 (RS1) : "À la demi-heure, la grande aiguille est positionnée sur le 6 ", la règle stratégique 2 (RS2) : «À la demi-heure, la petite aiguille se trouve entre 2 chiffres » et la règle stratégique 3 (RS3) : « Les 2 aiguilles avancent en même temps ». Mais pour autant ces trois règles stratégiques ne peuvent être véritablement mobilisées dans la mesure où les aiguilles sont désolidarisées. P2 prend en charge de positionner correctement les deux aiguilles pour afficher $1 \mathrm{~h} 30$.

Étape 2. Le professeur propose alors un nouveau Jeu d'Apprentissage qui devrait permettre aux élèves d'exercer les connaissances qui correspondent aux 3 règles stratégiques proposées dans le Jeu précédent. Il s'agit d'actionner les 2 aiguilles de l'horloge pour les faire passer de la position $1 \mathrm{~h} 30$ à la position $15 \mathrm{~h} 30$, soit le Jeu JA2 : «faire tourner les aiguilles sur le cadran de $1 \mathrm{~h} 30$ à $15 \mathrm{~h} 30$ ».

Ce Jeu prend appui sur le précédent, mais il est plus spécifique du savoir visé. Du point de vue des règles stratégiques, le professeur va s'appuyer sur les difficultés des élèves (toujours sur le mode essai-erreur-correction), pour donner à voir une quatrième règle 
(RS4) : «à l'heure pile, la grande aiguille est sur le 12 ». À partir de la situation, il finira par « donner " la règle stratégique (RS5) qui devrait permettre aux élèves d'établir la conversion $\mathrm{h} \mathrm{am} / \mathrm{h} \mathrm{pm}: \ll 14 \mathrm{~h}=12+2 »$. L'analyse montre que le professeur tente ainsi de faire jouer simultanément le Jeu JA2 et le Jeu JA3 «faire transformer une heure am en une heure pm selon la fonction $\mathrm{y}=\mathrm{x}+12 »$. Mais $\mathrm{P} 2$ prend complètement à sa charge l'énonciation didactique selon un quasi-monologue et dans une position surplombante de « correcteur/évaluateur».

Étape 3. Or, devant les difficultés rencontrées par les élèves, le professeur va renoncer. Il leur demande alors, sans doute pour ne pas perdre la «face» et ménager celle des élèves, quelle est la couleur de la petite aiguille, soit le Jeu JA4 : «faire produire une réponse concernant la couleur des aiguilles ». Ce Jeu est un Jeu très générique, qui ne mobilise aucun apprentissage et qui est déconnecté du Jeu Épistémique potentiellement visé par la situation d'aide. Les Jeux JA2 et JA3 disparaissent. Il se produit un véritable évanouissement des savoirs lié à une disparition de l'enjeu didactique. Il y a déconnection entre les Jeux d'Apprentissage et le Jeu Épistémique dans la mesure où les Jeux d'Apprentissage effectivement joués ne permettent pas l'actualisation du Jeu Épistémique. Le glissement de Jeu observé correspond en fait à un glissement d'objet : la notion d'heure ante meridiem et post meridiem disparaît dans le système sémiotique «pendule» au profit des aiguilles.

Pour ce professeur, comme pour P1, on observe un glissement de Jeu d'apprentissage lié à un déplacement du contrat didactique (contrat de résolution de problème à un contrat de description) et à un changement de focale concernant les objets du milieu (de la pendule aux aiguilles). Ce glissement se traduit par un double effet de contrat: d'une part par un effet Topaze (le professeur prend en charge les réponses aux questions qu'il pose) et d'autre part par un effet Jourdain (la couleur des aiguilles prend une valeur de production de savoir).

De même que chez $\mathrm{P} 1$, nous assistons à un glissement d'un Jeu d'Apprentissage spécifique (la conversion ante meridiem - post meridiem) à un Jeu d'Apprentissage très générique (produire une réponse orale). En revanche, pour ce qui concerne les règles, le professeur pose les règles définitoires qui permettent aux élèves de rentrer dans le Jeu, c'est-à-dire d'afficher des heures sur le cadran de l'horloge. Les élèves ne possédant pas les connaissances nécessaires et le milieu n'étant pas opératoire, ils ne peuvent néanmoins construire de leur propre mouvement aucune des règles stratégiques qui permettraient de jouer le Jeu Épistémique «Rendre les élèves capables de décomposer l'unité temporelle " jour » selon la forme : 1 jour $=24 \mathrm{~h}=12 \mathrm{~h} \mathrm{am}+12 \mathrm{~h} \mathrm{pm}$ ».

61 Du point de vue de l'évolution des objets, il apparaît que le temps court dont dispose le professeur va empêcher les élèves d'avoir une prise sur les objets matériels du milieu. Ces derniers restent alors la propriété du professeur dans la mesure où les élèves ne disposent pas de temps d'action et donc ne peuvent véritablement engager une expérience avec le savoir cristallisé dans l'objet "pendule». Par ailleurs, cet objet matériel ne prend jamais véritablement le statut d'objet mathématique. Il nous apparaît que si la pendule peine justement à prendre un statut mathématique, c'est en partie parce que les aiguilles sont désolidarisées: elles ne peuvent "avancer» ensemble. Cette inadéquation entre les objets matériels et les objectifs d'apprentissage tend à rendre le milieu de l'étude non opératoire.

Dans ce temps court, le professeur accumule pêle-mêle tous les objectifs mobilisés dans la séance collective ${ }^{16}$. Tout se passe comme si cette accumulation produisait une 
contraction en un même espace-temps de l'ensemble des objets mobilisés par le savoir en Jeu. De cette manière, $\mathrm{P} 1$ produit pour les élèves une synthèse qui pourrait prendre la forme d'une sorte d'institutionnalisation des connaissances (Brousseau, 2003), mais qui dans les faits n'en est pas une car il n'y a pas « reconnaissance de la validité et de l'utilité de cette nouvelle connaissance par les élèves" (ibid). On peut y voir alors, ce que nous appellerons une sorte de leurre.

Tableau 5 : Éléments d'épistémologie pratique du professeur P2

\begin{tabular}{|c|c|}
\hline Verbatim & Éléments de l'épistémologie pratique de P2 \\
\hline \multicolumn{2}{|l|}{ Rapport de P2 à la difficulté scolaire } \\
\hline $\begin{array}{l}\text { Conserver un petit moment avec les enfants qui } \\
\text { n'y arrivent pas et essayer d'expliquer encore. } \\
\text { Je vais prendre encore du temps avec eux. }\end{array}$ & $\begin{array}{l}\text {-La difficulté scolaire se traite par l'explication } \\
\text { réitérée en petit groupe homogène. } \\
\text {-Donner plus de temps est la solution pour traiter la } \\
\text { difficulté scolaire. }\end{array}$ \\
\hline $\begin{array}{l}\text { Je vais relancer sur la bonne piste les élèves qui } \\
\text { ont le plus de difficulté. }\end{array}$ & $\begin{array}{l}\text {-La difficulté scolaire provient de l'activation de } \\
\text { «mauvaises » procédures ou de choix de données } \\
\text { non pertinentes. Aider les élèves, c'est les aiguiller } \\
\text { dans la bonne direction. }\end{array}$ \\
\hline $\begin{array}{l}\text { Ce sont des enfants volontaires qui veulent } \\
\text { progresser } \\
\text { Il faut que les élèves aient au moins réussi ce } \\
\text { minimum. }\end{array}$ & $\begin{array}{l}\text {-L'aide passe par le maintien de l'exigence d'un } \\
\text { contrat de réussite minimum. }\end{array}$ \\
\hline \multicolumn{2}{|l|}{ Rapport de P2 au savoir enseigné et à l'apprentissage } \\
\hline $\begin{array}{l}\text { J'ai fait confiance au manuel et je pense que la } \\
\text { formulation est un peu ambiguë. } \\
\text { Passer des heures du matin à celles de l'après- } \\
\text { midi,je pensais que ça aurait été plus facile. } \\
\text { Dans la préparation je fais attention à avoir des } \\
\text { moments collectifs et individuels. }\end{array}$ & $\begin{array}{l}\text {-L'analyse des contenus de savoirs est prise en } \\
\text { charge par le manuel. } \\
\text { - Le professeur prend en charge les aspects } \\
\text { organisationnels. }\end{array}$ \\
\hline $\begin{array}{l}\text { J'essaie de dire le vrai et d'institutionnaliser et de } \\
\text { reformuler et c'est à la fin du troisième tiers de } \\
\text { l'exercice où ils doivent normalement réussir à } \\
\text { faire } \\
\text { Ils ont tous fait, ils ont tous été actifs et ils ont } \\
\text { tous fait en très peu de temps. } \\
\text { S'ils réussissent à faire dans le temps, c'est un } \\
\text { défi par rapport à soi-même. }\end{array}$ & $\begin{array}{l}\text {-C'est la diffusion des procédures expertes dans la } \\
\text { classe qui favorise la réussite de tous les élèves. } \\
\text {-Etre actif et rapide est, pour le professeur, une } \\
\text { manifestation de la réussite. }\end{array}$ \\
\hline
\end{tabular}

63 Aider pour P2 signifie (ré)-expliquer, répéter, sans réaménagement de la situation initiale, à l'identique de la situation développée en classe. Pourtant, il semblerait que c'est justement pour ramener ces 2 élèves dans le temps didactique du collectif de la classe, que $\mathrm{P} 2$ ne produit pas de réaménagement du milieu par rapport à la situation initiale. 

d'épistémologie pratique, de définir le format qui organise, pour P2, les gestes de l'aide :

- Reprendre à l'identique des situations échouées en classe pour remettre "à niveau » les élèves en difficulté.

- Organiser l'aide sur des temps courts, dans l'après-coup de la séance, avec de très petits groupes homogènes « faibles » dirigés par le professeur.

- Montrer et diffuser les «bonnes manières» d'agir sur le milieu, dans le but d'amener les élèves à faire « comme le professeur ».

- Conduire les élèves vers la bonne réponse en corrigeant systématiquement leurs erreurs et en leur donnant la réponse juste.

\section{Ré-information de l'analyse didactique}

Dans cette analyse comparative des deux cas didactiques, nous allons nous employer à mettre au jour certaines convergences et, sur la base de celles-ci, isoler certaines singularités qui apparaissent utiles à la compréhension de l'action conjointe. L'identification de ces éléments de convergence et de singularité pourrait nous permettre de fonder quelques-unes des déterminations des gestes de l'aide ordinaire à l'école primaire, mais aussi les caractéristiques de ce que nous avons d'ores et déjà nommé « un cas didactique limite». 


\section{Les déterminations des gestes de l'aide}

\section{Le comportement du professeur face à l'échec}

Concernant le comportement des deux professeurs face à l'échec, nous avons rapproché nos observations de celles de Brousseau $(2006)^{17}$. Il s'avère que nous partageons avec lui les 4 points suivants que nous complèterons systématiquement avec les résultats de nos analyses.

- L'allongement illimité du temps d'enseignement (ibid) et la concentration sur les savoirs de bas niveau taxonomique (ibid) : face à l'échec, l'enseignant reprend l'ensemble des objets d'apprentissage afin de tendre vers la forme de savoir visée. Dans nos deux cas, P1 et P2 ont tendance à orienter les élèves vers des procédures de plus bas niveaux qui reviennent à simplifier la tâche, mais qui, pour autant, sont encore plus chronophages.

- La définition de l'enseignement (ibid): les professeurs répondent à l'échec par des formes d'organisation prônant une individualisation de l'aide et selon des groupes de niveau. Toutefois, nous ajoutons que, comme ce temps consacré à l'aide en groupe «homogène faible » n'est que ponctuel, nous observons plusieurs choix : soit, comme nous le voyons chez P2 «aller très vite » pendant des temps courts d'aide (contraction du temps), soit comme chez P1, travailler via des plans de travail qui autorise une dilatation du temps dévolu à l'aide.

- La fragmentation du savoir: Brousseau (ibid) souligne que tout «échec" amène à une décomposition en savoirs plus élémentaires. Ainsi, pour l'élève, les liens entre les savoirs décomposés sont alors de plus en plus difficiles à établir. P1, en modifiant le milieu de manière successive et multiple, exige des élèves un nombre important de tâches et P2, en voulant conserver l'ensemble des objectifs d'apprentissage travaillés lors de la séance collective, finit par produire une simple juxtaposition des tâches.

Nos analyses de ces situations d'aide montrent d'autres points de convergence dans les choix réalisés par les deux professeurs et qui nous semblent traduire, cette fois, certains obstacles à la mise en place de gestes de l'aide.

\section{Des obstacles à la mise en place de gestes de l'aide}

71 Nos analyses ont rendu apparents quatre types d'obstacles : un ancrage épistémique faible et une entrée par le générique, des milieux peu opératoires, un guidage vers la production d'une trace comme format scolaire obligatoire, une reprise de la manière d'enseigner ordinaire.

\section{Un ancrage épistémique faible et une entrée par le générique}

Dans les entretiens des deux professeurs, très peu d'énoncés se réfèrent explicitement aux savoirs en jeu et quand ils y font allusion, les savoirs ne sont pas interrogés.

P1: Un trait rouge, c'est pas compliqué; C'est une situation qui est simple à mettre en œuvre.

P2 : Je pensais que ça aurait été plus facile.

P1 et P2 disent ne pas réussir à sérier ce qui fait difficulté pour les élèves, Nous référons cette incompréhension à un défaut de prise d'indices dans les actions et comportements des élèves par le professeur. Pour savoir où et quoi observer, encore faut-il avoir une idée précise de l'objet de l'enquête. Or, l'ancrage épistémique des

Éducation et didactique, 5-3 | 2011 
professeurs et la connaissance des obstacles que pourraient rencontrer leurs élèves ne fait pas partie des moyens dont ces professeurs s'outillent pour accéder à plus de compréhension des causes de la difficulté ${ }^{18}$. L'élève, dans les situations d'aide étudiées, semblerait plus facilement appréhendé comme un élève générique (cas des "aiguilles ») ou comme un élève singulier (cas des "rectangles») et finalement peu souvent comme un élève épistémique ${ }^{19}$.

\section{Des milieux peu opératoires}

Nous avons observé que les Jeux annoncés n'ont pu être joués ; soit que le professeur se substitue aux élèves (P2), soit qu'il peine à leur faire percevoir les règles définitoires du Jeu (P1), soit que certains objets nécessaires à la mise en œuvre du Jeu sont absents du milieu (P2). Lorsque les Jeux annoncés ne peuvent être joués, nous observons alors un changement de focale vers d'autres objets, épistémiquement moins denses. Ces "glissements de Jeux », c'est-à-dire une remontée vers des Jeux moins spécifiques, voire génériques, s'accompagnent d'un affaiblissement de l'enjeu didactique. Toutefois, il est important de noter ici que le glissement de Jeu d'Apprentissage ne peut en aucun cas être considéré en soi comme un dysfonctionnement de la relation enseignementapprentissage : c'est son analyse dans le contexte de la situation (de savoir) qui permet au chercheur d'évaluer son effet sur l'apprentissage des élèves.

\section{Un guidage vers la production d'une trace comme format scolaire obligatoire}

77 Face à cette non-compréhension de la difficulté, et sans doute dans un souci de réassurance et de restauration de la place de chaque élève au sein du collectif de la classe, P1 et P2 guident les élèves vers la production d'une trace (dessiner un rectangle pour P1 et énoncer la couleur des aiguilles pour P2). Cette trace, bien que déconnectée de l'objectif d'apprentissage initial, peut alors attester de la "réussite » d'une tâche, même s'il s'agit, dans les deux cas, d'un leurre en ce sens que le lien didactique se maintient, mais de manière artificielle. Tout se passe comme si conserver ce lien didactique maintenait les élèves dans le temps didactique de la classe.

\section{Une reprise de la manière d'enseigner ordinaire}

Il nous apparaît également que pour ces deux professeurs, c'est leur manière habituelle de faire la classe qui va organiser leur pratique d'aide. La spécificité de la difficulté n'est pas considérée. Dans le cas des aiguilles, P2 réitère, mais de façon plus rapide, la façon dont il fait une «leçon »: mise en situation, reformulation de la consigne, retour à l'exercice, institutionnalisation, retour à l'exercice. Dans le cas des «rectangles », P1 tente de s'appuyer sur les élèves pour faire avancer le temps didactique, comme il le fait habituellement dans l'encadrement des groupes d'élèves hétérogènes quand ils travaillent avec leurs "plans de travail individuel». Sauf que dans ce cas, le groupe étant un groupe homogène "faible », cet appui n'est pas possible.

Ce complément d'analyse nous montre que les gestes du professeur dévolus à l'aide sont déterminés par des épistémologies singulières qui concernent :

- le rapport du professeur à l'enseignement avec une épistémologie de l'obstacle pour P1 où il s'agit de faire expliciter la difficulté et une épistémologie du "juste/faux » pour P2 où il

s'agit de faire imiter et répéter la bonne procédure, 
- le rapport du professeur à la forme scolaire avec, pour P1 l'idée de la classe hétérogène avec des parcours différenciés et pour P2 de la classe homogène avec un parcours unique où l'écart avec l'avancée collective du temps didactique ne peut être trop longtemps maintenu.

- le rapport du professeur à l'élève en difficulté avec pour P1, une posture de sollicitude et pour P2, une posture d'entraîneur au sens de « celui qui entraîne les élèves à agir ».

Pour autant, ces épistémologies différentes donnent à voir, dans les 2 cas, un guidage «pas à pas » du professeur. Ce guidage n'autorise pas les élèves à vivre de véritables expériences avec les objets de savoir de la situation et ces derniers ne modifient pas in fine leur rapport au savoir en jeu.

81 Tout se passe alors comme si ces formats de l'aide jouaient le rôle d'allant de soi, au risque de ne plus être réinterrogés par les enseignants. L'action conjointe, au lieu d'être chronogène (générer des apprentissages par l'avancée du temps didactique), devient chronophage. En voulant donner plus de temps aux élèves, il se produit en fait l'effet inverse : une « perte » de temps (Chopin, 2008, 2010).

\section{Caractérisation des « cas didactiques limite »}

Nous avons sélectionné dans nos analyses les éléments qui nous paraissent les plus significatifs pour expliquer l'évanouissement des savoirs auquel nous assistons dans chacun des deux cas. Cet évanouissement des savoirs est en soi la première caractéristique d'un « cas didactique limite ».

Nous avons d'abord recherché les éléments convergents relatifs à cet évanouissement, pour ensuite les décliner en éléments plus singuliers, selon les deux cas : celui des "aiguilles" et celui des "rectangles». Le tableau qui suit reprend de manière synoptique l'ensemble de ces éléments. Cette exposition synoptique devrait rendre plus aisée l'analyse comparative.

Tableau 6 : Analyse comparative des 2 cas - Convergences et singularités

\begin{tabular}{|c|c|c|}
\hline \multirow{2}{*}{$\begin{array}{l}\text { Éléments convergents P1 } \\
\text { et P2 }\end{array}$} & \multicolumn{2}{|l|}{ Éléments singuliers } \\
\hline & Cas « des aiguilles » P2 & Cas « des rectangles" \\
\hline $\begin{array}{l}\text { Le milieu matériel ne } \\
\text { s'objective pas pour les } \\
\text { élèves en } \quad \text { milieu } \\
\text { mathématique. }\end{array}$ & $\begin{array}{l}\text { Milieu matériel (la pendule) qui } \\
\text { s'assimile à un leurre de milieu } \\
\text { mathématique. }\end{array}$ & $\begin{array}{l}\text { Glissement du milieu } \\
\text { mathématique (le quadrillage avec } \\
\text { la stratégie multiplicative) au } \\
\text { milieu matériel (l'objet } \\
\text { « rectangle » à découper). }\end{array}$ \\
\hline $\begin{array}{l}\text { Les élèves n'élaborent pas } \\
\text { de règles stratégiques qui } \\
\text { leur permettraient de } \\
\text { "gagner au Jeu », c'est-à- } \\
\text { dire d'apprendre. }\end{array}$ & $\begin{array}{l}\text { effet Topaze : P2 «souffle la } \\
\text { réponse » aux élèves } \\
\text { effet Jourdain : P2 atteste d'un } \\
\text { savoir là où il ne s'en est pas } \\
\text { produit (la couleur des aiguilles). }\end{array}$ & $\begin{array}{l}\text { effet Jourdain : P1 atteste d'un } \\
\text { savoir là où il ne s'en est pas } \\
\text { produit (le découpage d'un } \\
\text { rectangle). }\end{array}$ \\
\hline
\end{tabular}




\begin{tabular}{|c|c|c|}
\hline $\begin{array}{l}\text { Par le monopole de la } \\
\text { parole, les deux professeurs } \\
\text { ne permettent pas aux } \\
\text { élèves de se confronter } \\
\text { directement aux objets de } \\
\text { savoir. } \\
\text { Les deux professeurs } \\
\text { occupent une position } \\
\text { surplombante par un } \\
\text { guidage «pas à pas». }\end{array}$ & $\begin{array}{l}\text { Les élèves sont guidés vers la } \\
\text { bonne réponse par la correction } \\
\text { immédiate de P2. }\end{array}$ & $\begin{array}{l}\text { Les élèves, par un guidage serré de } \\
\text { P1, sont absorbés dans des } \\
\text { activités ponctuelles sans lien } \\
\text { entre elles dont le professeur tient } \\
\text { à lui seul les tenants et les } \\
\text { aboutissants. }\end{array}$ \\
\hline $\begin{array}{l}\text { Les professeurs tentent de } \\
\text { rapprocher les élèves en } \\
\text { difficulté du temps } \\
\text { didactique de la classe. }\end{array}$ & $\begin{array}{l}\text { Contraction du temps de } \\
\text { l'apprentissage et regroupement } \\
\text { des objets de savoir sur le temps } \\
\text { très court de l'aide. } \\
\text { Surinvestissement par P2 des } \\
\text { objets de savoir de la situation } \\
\text { (mobilisation des mêmes objets } \\
\text { que dans la situation échouée). }\end{array}$ & $\begin{array}{l}\text { Dilatation du temps de } \\
\text { l'apprentissage et éparpillement } \\
\text { des objets de savoir. } \\
\text { Sousinvestissement par P1 des } \\
\text { objets de savoir de la situation (le } \\
\text { quadrillage n'est pas investi). }\end{array}$ \\
\hline $\begin{array}{l}\text { Dans les } 2 \text { cas, nous } \\
\text { observons des glissements } \\
\text { de Jeux d'Apprentissage } \\
\text { avec affaiblissement de } \\
\text { l'enjeu didactique initial. }\end{array}$ & $\begin{array}{l}\text { Simulacre de } \\
\text { l'institutionnalisation: P2 } \\
\text { institue les règles stratégiques } \\
\text { qu'il a proposées lui-même. }\end{array}$ & $\begin{array}{l}\text { Simulacre de la régulation: } \\
\text { P1 sollicite (en vain) les élèves } \\
\text { pour qu'elles mettent en mots ce } \\
\text { qu'elles ne comprennent pas. }\end{array}$ \\
\hline
\end{tabular}

Afin de construire, à partir de ces éléments d'analyse, les premières caractéristiques de ce que nous avons appelé " un cas didactique limite ", nous nous sommes appuyées sur les outils de la TACD, tels que développés dans ce travail. Nous pouvons ainsi définir ce qu'est un "cas didactique limite» du point de vue de ces descripteurs de l'action conjointe que sont les notions de système $\mathrm{JA} / \mathrm{JE}$, de règles définitoires et stratégiques et de glissement ou de disparition de Jeu d'Apprentissage. Ainsi, dans un cas didactique limite :

- Il y a déconnexion entre les Jeux d'Apprentissage effectivement joués et les Jeux Epistémiques visés.

- Le milieu de l'étude est peu défini par les professeurs, les objets valent pour eux-mêmes : ce qui génère de la part des élèves des interprétations très personnelles et qui peuvent s'avérer conflictuelles avec les attendus de l'apprentissage visé. Les règles définitoires ne sont pas identifiées. Aussi les élèves ne savent-ils pas comment jouer au Jeu d'Apprentissage, donc a fortiori comment faire pour y gagner. Le Professeur n'a alors d'autre solution que de proposer lui-même les règles stratégiques et d'assumer la responsabilité de la situation, ou bien de provoquer des glissements successifs de Jeux d'Apprentissage.

- Ces glissements de Jeux d'Apprentissage donnent lieu à des disparitions de JA ou parfois à des transformations en "Jeux fantôme", c'est-à-dire en des Jeux qui ne conservent que l'apparence du JA spécifique initialement attendu.

- Ces glissements se traduisent par des effets de contrat didactique (effet Topaze et/ou effet Jourdain) qui, à l'extrême, peuvent conduire élèves et professeurs à «tricher» au Jeu en attestant d'un apprentissage, là où il ne s'en est pas produit.

- le professeur occupe une position surplombante (ou faussement basse) qui conditionne un guidage très serré de l'activité des élèves : ces derniers ne peuvent pas se " prendre au Jeu » 
dans la mesure où les enjeux de la situation restent opaques. Ils n'ont pas accès aux finalités de leurs actions.

- La priorité est donnée au maintien du lien didactique afin de préserver la place et la face du professeur comme des élèves. Les transactions didactiques attendues (dans lesquelles l'objet transactionnel est le savoir) sont remplacées par des interactions peu denses épistémiquement qui produisent des traces d'activité stéréotypées. point de vue de l'action conjointe et d'associer à cette notion celles de stratégie-recours et de zone-refuge. La validation du caractère opératoire de ce système descripteur de la relation d'enseignement/apprentissage quand elle se situe aux frontières du didactique, est un objectif de nos prochains travaux. Nous inaugurons ainsi, dans le cadre de notre méthodologie de l'étude de cas, une première famille, celle des " "cas didactiques limite" " dont les premières caractéristiques, ici dégagées, seront complétées, affinées, voire amendées, lors de la confrontation avec de nouveaux cas, dans des contextes similaires ${ }^{20}$. Contribuer à la construction de cette première famille était là un des objectifs de cette étude. En effet, selon les termes de Passeron et Revel (2005, p. 9), nous avons tenté de procéder dans cet article à une "exploration et à l'approfondissement des propriétés d'une singularité accessible à l'observation» dans le but d'en extraire « une argumentation de portée plus générale, dont les conclusions pourront être réutilisées pour fonder d'autres intelligibilités ou justifier d'autres décisions». C'est en prise avec cette orientation qu'ont été construits nos deux cas prototypiques (Livet, 2005) : « Les rectangles » et « les aiguilles».

\section{Conclusion}

Nous avons montré, dans cet article, que l'aide en classe consiste surtout, pour P1 et P2, en une organisation en petit groupe dans la classe. L'intention des deux professeurs est que les élèves rattrapent ainsi le temps didactique collectif, soit selon une accélération 
du temps (reprise des objectifs collectifs sur un temps court pour P2), soit selon une dilatation de ce temps (avec reprise d'objets antérieurs non stabilisés pour P1), mais qui aboutissent, dans l'un et l'autre cas, à un évanouissement des savoirs.

Il nous apparaît que ce n'est pas le travail en petit groupe qui est contre productif en lui-même, mais plutôt la non prise en compte des aspects didactiques par les deux professeurs. En effet, au lieu d'un travail effectif de réaménagement du milieu qui permettrait de réduire la distance des élèves au savoir visé et de maintenir la connexion aux objectifs d'apprentissage, nous observons des gestes stéréotypés qui pourraient s'apparenter à une certaine forme de doxa qui traverse le métier. Cette doxa inhérente à l'aide consisterait à mettre les élèves en groupe "homogène faible ", à simplifier, voire éparpiller les tâches, à faire réussir les élèves à tout prix au travers de la production de traces, en partie déconnectées des enjeux de savoir. Ces gestes stéréotypés ne semblent pas permettre aux élèves de rattraper le temps collectif. Dans les deux contextes, l'aide in fine, maintient ou restaure la «face » ou la «place» des acteurs.

Ces premières déterminations des gestes de l'aide ordinaire devraient nous permettre de nous orienter maintenant vers l'étude de l'action conjointe dans les situations d'aide personnalisée actuellement mises en place depuis 2008 sous l'impulsion de plusieurs circulaires $^{21}$. La demande institutionnelle et sociale semble en effet actuellement se focaliser sur le "traitement » de la difficulté d'apprentissage dans la mesure où ces difficultés pourraient avoir partie liée avec l'accroissement des inégalités scolaires révélé par les résultats des dernières évaluations PISA (2009) ${ }^{22}$. L'analyse des textes officiels montre des attentes relatives à un « renouveau de la forme scolaire » via une demande accrue d'individualisation des aides. Il nous semble maintenant opportun d'aller voir si ces pratiques d'aides personnalisées, vécues dans un autre contexte, vont se trouver reconfigurées et s'émanciper des formes stéréotypées mises au jour dans cette étude (Toullec-Théry, Marlot, à paraître). Ainsi, il serait peut-être envisageable à partir de ces dispositifs d'aide personnalisée, de repenser l'aide ordinaire dispensée en classe et inversement.

\section{BIBLIOGRAPHIE}

Amade-Escot, C. \& Venturini, P. (2009). Le milieu didactique : d'une étude empirique en contexte difficile à une réflexion sur le concept. Éducation \& didactique, 3 (1), 7-43.

Auriac, E. (2007). Quels indicateurs et unités d'analyse privilégier pour progresser dans l'étude des discours scolaires? In A. Specogna (Ed). Enseigner dans l'interaction. Paris : L'Harmattan, 33-56. Brousseau, G. (1998). Théorie des situations didactiques. Grenoble : La pensée Sauvage.

Brousseau, G. (2003). Glossaire de quelques concepts de la théorie des situations didactiques en mathématiques. http://daest.pagesperso-orange.fr/guy-brousseau/textes/Glossaire_Brousseau. pdf 
Brousseau, G. (2006). Epistemologia e didattica della matematica. La matematica e la sua didattica, 4 , 4-57.

Bru, M. \& Altet, M. \& Blanchard-Laville, C. (2004). À la recherche des processus caractéristiques des pratiques enseignantes dans leurs rapports aux apprentissages. Revue française de pédagogie, 148, 75-87.

Chevallard, Y. (1992). Concepts fondamentaux de la didactique : perspectives apportées par une approche anthropologique, in Recherches en Didactique des mathématiques, vol. 12, $\mathrm{n}^{\circ}$ 1, 73-112.

Chopin, M.-P. (2010). Les usages du « temps » dans les recherches sur l'enseignement, Revue française de pédagogie, 170.

Chopin, M.-P. (2008). La visibilité didactique : un milieu pour l'action du professeur. Présentation d'un concept pour l'étude des pratiques d'enseignement, Éducation et didactique, vol. 2, n² 2, 63-79.

Chopin, M.-P. (2006). Temps d'enseignement et temps didactique Approche didactique de la question du temps dans l'enseignement des mathématiques au cycle 3 de l'école élémentaire. Carrefours de l'éducation, 21, 53-71.

D’Amore, B., Fandino Pinilla, M.I., Marazzani, I., Santi, G. \& Sbaragli, S. (2008). Le rôle de l'épistémologie de l'enseignant dans les pratiques d'enseignement. Communication présentée au colloque international « les didactiques et leurs rapports à l'enseignement et à la formation ». Bordeaux. France. Septembre.

Develay, M. (1997). Origines, malentendus et spécificités de la didactique. Revue Française de Pédagogie, 120, 59-66.

Ginzburg, C. (2001). À distance. Neuf essais sur le point de vue en histoire. Paris : Gallimard.

Goffman, E. (1973). La mise en scène de la vie quotidienne, Les relations en public. Tome 2, Paris : Minuit.

Goffman, E. (1991). Les cadres de l'expérience. Paris : Les Éditions de Minuit.

Gruson, B. (2009). Étude de la dialectique contrat-milieu dans l'enseignement-apprentissage de l'anglais en CM2 et en $6^{\mathrm{e}}$. Revue Suisse des Sciences de l'éducation. Vol. 3.

Livet, P. (2005). Les diverses formes de raisonnement par cas. In J-C Passeron \& J. Revel (Eds.), Penser par cas, 229-254. Paris : Éditions de l'EHESS.

Marlot, C. (2008). Caractérisation des transactions didactiques. Deux études de ces à l'école élémentaire en découverte du monde vivant. Thèse de doctorat, Université Rennes 2.

Marlot, C. \& Toullec-Théry, M. (2009). Fading knowledge and « learning game shifting » : a comparatist approach in two scientific disciplines at primary school (first grade). Communication in The European Conference on Educational Research (ECER) « Theory and evidences in european educational research ». Vienna. Austria. September.

Marlot, C. (2009). Glissement de Jeu d'Apprentissage scientifiques et épistémologie pratique de professeurs au CP. ASTER $n^{\circ}$ 49, 109-136. Enseignements scientifiques et techniques dans la scolarité obligatoire. Paris : INRP.

Marlot, C. (2010). Généricité et spécificité des transactions didactiques en découverte du monde vivant au cycle 2 : épistémologie pratique de la « polyvalence » chez un professeur. Colloque international «Spécificités et diversité des interactions didactiques : disciplines, finalités, contextes ». 24-26 juin. Lyon UMR ICAR, ENS-LSH, INRP, EA DILTEC. 
Marlot, C. (à paraître). Glissement de Jeu d'Apprentissage et capital d'adéquation des élèves : une étude de cas à l'école élémentaire en classe de sciences. In B. Gruson, D. Forest \& M. Loquet (Eds.) Jeux de savoir. Rennes : Presses universitaires de Rennes.

Mercier, A. Schubauer-Leoni, M.-L., Sensevy, G. (Eds) (2002). Vers une didactique comparée. Revue Française de pédagogie, 141, 5-16. Paris : INRP.

Passeron, J.-C. \& Revel, J. (2005). Penser par cas. Paris : Éditions de l'EHESS.

Renaut, A. (2002). La libération des enfants. Contribution philosophique. Paris : Bayard.

Santini, Jérôme (2009). Caractérisation de l'élaboration conjointe de la compréhension conceptuelle et des performances associées. Volcans et séismes au Cours Moyen. Thèse de doctorat, Université Rennes 2.

Schubauer-Leoni, M.-L., Leutenegger F., Ligozat \& F., Fluckiger, A. (2007). Un modèle d'action conjointe professeur-élèves : les phénomènes didactiques qu'il peut/doit traiter. In G. Sensevy \& A. Mercier (Eds.), Agir ensemble : l'action didactique conjointe du professeur et des élèves. Rennes : PUR. Sensevy, G., Mercier, A., Schubauer-Leoni, M.-L. (2000). Vers un modèle de l'action didactique du professeur. À propos de la Course à 20, Recherches en Didactique des mathématiques, 20 (3), 263-304.

Sensevy, G. (2001). Théories de l'action et action du professeur. In J.-M. Baudouin J. Friedrich (Eds.), Théories de l'action et éducation (p. 203-224). Bruxelles : De Boeck.

Sensevy, G. (2002). Représentations et action didactique. L'année de la recherche en sciences de l'éducation, 67-89.

Sensevy, G. (2006 a). Esquisse d'une pragmatique didactique. Communication présentée au séminaire IVDA. France. Lyon. 2006.

Sensevy, G. (2006 b). L'action didactique. Éléments de théorisation. Revue suisse des sciences de l'éducation, 2, 205-226.

Sensevy, G. \& Mercier, A. (2007). Agir ensemble : l'action didactique conjointe. In G. Sensevy \& A. Mercier (Eds.), Agir ensemble : l'action didactique conjointe du professeur et des élèves. Rennes : PUR.

Sensevy, G. (2007). Des catégories pour décrire et comprendre l'action didactique. In G. Sensevy \& A. Mercier (Eds.), Agir ensemble : L'action didactique conjointe du professeur et des élèves (p. 13-34). Rennes : Presses Universitaires de Rennes (PUR).

Sensevy, G., Maurice, J.-J., Clanet, J. \& Murillo, A. (2008). La différenciation didactique passive : un essai de définition et d'illustration. Les Dossiers des Sciences de l'Éducation, 20, 105-122.

Sensevy, G. (2010). Les formes de l'intention didactique : communauté de pratique, institution, collectif de pensée ? In G. Gueudet, \& L. Trouche (Eds.), Ressources vives. Rennes : Presses Universitaires de Rennes.

Sensevy, G. (2011). Le sens du savoir. Éléments pour une théorie de l'action conjointe en didactique. Bruxelles : De Boeck.

Toullec-Théry, M. (2006) Aider les élèves « peu performants » en mathématiques à l'école primaire: quelles actions des professeurs? Étude in situ de professeurs des écoles de classes ordinaires et de maîtres spécialisés à dominante pédagogique. Thèse de doctorat, Université Rennes 2.

Toullec-Théry, M. (2009). Practical ingenuity of specialized teachers who work with « underperforming » pupils. Communication in The european Conference on Educational Research $(E C E R)$ «Theory and evidences in european educational research» Vienna. Austria. September. 
Toullec-Théry, M. (à paraître). Dans le regroupement d'adaptation, existe-t-il des régimes d'attention spécifiques aux maîtres spécialisés à dominante pédagogique ? In B. Gruson, D. Forest \& M. Loquet (Eds.) Jeux de savoir. Rennes : Presses universitaires de Rennes.

Toullec-Théry, M., Marlot, C. (à paraître). L'aide ordinaire en classe et dans les dispositifs d'Aide Personnalisée à l'école primaire : une approche comparatiste en didactique. In C. Marlot \& M. Toullec-Théry (coord.), Diversification des parcours des élèves : pratiques enseignantes et organisations scolaires en question. Recherche En Éducation. Hors série $n^{\circ} 4$.

\section{NOTES}

1. Parler de transaction plutôt que d'interaction nous permet, dans le cadre de cette théorie, de situer d'emblée notre focale à la fois sur le savoir en jeu et sur la façon dont les acteurs co-élaborent ce savoir.

2. Pour autant, nous n'excluons pas l'idée d'une forte corrélation entre réussite et apprentissage et il reste entendu que quel que soit le degré ou la nature des attentes, le professeur est intrinsèquement animé par l'intention d'enseigner et de "faire apprendre ".

3. D'après Brousseau (1998), le milieu est l'environnement spécifique d'un savoir ou d'un de ses aspects. Dans notre travail, nous ferons référence à la définition du milieu de Chevallard (1992) comme l'ensemble des objets matériels et symboliques sur lesquels agissent le professeur et les élèves.

4. Nous appelons «milieu transactionnel le milieu constitué par les transactions professeur-élèves, qui détermine en partie la manière dont les élèves et le professeur reçoivent et perçoivent les éléments du milieu matériel ou objectif » (Sensevy, 2010).

5. Ces théories peuvent prendre leur origine dans les rapports de l'individu au savoir enseigné, à l'apprentissage, au développement de l'enfant, à la difficulté scolaire...

6. Par exemple, une fois acquis l'apprentissage de la lecture en tant que Jeu spécifique, le Jeu "lire l'énoncé d'un problème » (dans le contexte d'un autre apprentissage) ne représente plus un Jeu d'Apprentissage spécifique ; l'enjeu de la séance est ailleurs. Le Jeu «Lire l'énoncé du problème » représente alors un Jeu très générique qui vient en appui au Jeu spécifique lié lui, à la résolution même du problème posé : il est nécessaire, mais non suffisant pour réaliser l'apprentissage visé.

7. Toutefois dans la classe, ces deux catégories de règles sont extrêmement liées et il est difficile d'en tracer la frontière. Cette différence n'est pas sans nous rappeler celle établie par E. GOFFMAN $(1991,33)$ entre deux catégories de règles, celles du jeu de dames et celles de la circulation routière : "Les premières permettent de comprendre l'objectif que les joueurs cherchent à atteindre, alors que les secondes ne nous disent ni où nous devons aller ni pourquoi nous devrions nous déplacer et se contentent de nous indiquer ce qu'il faut faire si l'on tient à se déplacer. » On se rend bien compte qu'il y a du définitoire dans la règle stratégique.

8. Le raisonnement à partir de prototype s'appuie sur un exemple concret - le prototype - «sur lequel on peut découvrir de nouvelles propriétés. Il permet de faire des inférences normales par similarité avec d'autres cas sans qu'on s'émeuve du fait que ces cas ne présentent pas de similarité sur tous les points avec le prototype. Le prototype permet de rassembler des cas par similarité partielle. » (Livet, 2005, p 236). 
9. Le travail des élèves est organisé à partir d'un outil caractéristique des techniques utilisées en pédagogie « Freinet » : le plan de travail.

10. La consigne du manuel véhicule beaucoup d'implicite. Elle aurait pu s'exprimer ainsi : "En utilisant un seul segment et en se servant du quadrillage, délimite un rectangle de 444 (48) carreaux. »

11. Le système de numérotation des différents Jeux a évolué depuis nos derniers travaux (Marlot, 2008 ; 2009). Pour une meilleure visibilité de la dynamique et du fonctionnement en système des différents Jeux d'Apprentissage, ces derniers sont maintenant numérotés dans l'ordre de leur apparition, de JA1 à JA n + 1 .

12. $t d p=$ tour de parole.

13. Le Jeu d'Apprentissage JA2 pourrait apparaître, en première analyse, comme un Jeu d'Apprentissage générique. Nous le considérons pourtant ici comme un jeu d'apprentissage spécifique dans la mesure où « exprimer l'écart entre la consigne et sa réalisation » revient in fine à faire jouer le Jeu Épistémique, c'est-à-dire à rendre les élèves capables de produire une stratégie multiplicative (en appui sur la réitération de l'addition).

14. Le quadrillage peut alors, par exemple, renvoyer à d'autres actions comme celles de comptage de carreaux pour calculer l'aire d'un rectangle ; de manipulation d'un tableau à double entrée pour trier, ranger, procéder à une catégorisation; de déplacement sur un quadrillage pour apprécier l'espace, se situer; de construction de gabarits/blocs pour effectuer des opérations de calcul.

15. «La sollicitude désigne ce sentiment de responsabilité que nous éprouvons pour autrui, y compris en l'absence de toute revendication de sa part, au nom d'un quelconque droit: elle conduit à lui apporter, dans la diversité des situations où l'inquiétude, la souffrance, le désespoir le rendent particulièrement vulnérable, ce que nous désignons avec le plus de simplicité et de précision quand nous parlons d'un soutien moral » (Renaut, 2002).

16. Rappelons les trois principaux objectifs : 1- Lire et/ou afficher des heures sur des horloges analogiques. 2- Positionner la grande aiguille pour indiquer quart d'heure et demi-heure. 3- Calculer des durées. Le quatrième objectif représentait le savoir nouveau et donc l'objectif d'apprentissage ciblé pour cette séance 4- Transformer l'heure ante meridiem en heure post meridiem.

17. Cité par d'Amore et al. (2008).

18. Ce phénomène est identifié à travers le concept de "visibilité didactique " pour le professeur dans les travaux de Chopin (2008).

19. Dans les instructions officielles, il est question de l'élève épistémique, c'est-à-dire le destinataire idéal des contenus de savoirs organisés en programme (Develay, 1997).

20. C'est-à-dire des contextes scolaires où une intention d'enseigner est affichée, que ce soit dans le collectif de la classe ou dans celui de l'intervention du professeur auprès de groupes d'aide.

21. Circ. 2008-042 du 4/04/2008 Préparation de la rentrée 2008; Circ. 2008-082 du $5 / 06 / 2008$, aménagement du temps scolaire, organisation du temps d'enseignement scolaire et l'aide personnalisée dans le $1^{\text {er }}$ degré; Circ. 2008-105, du 6/08/2008, obligations de service des personnels enseignants du $1^{\text {er }}$ degré ; Circ. 2010-38 du $18 / 03 / 2010$, Préparation de la rentrée, dont personnalisation des parcours scolaires. 


\section{RÉSUMÉS}

Ce travail se situe dans le cadre de l'approche comparatiste en didactique et s'appuie sur le système de description Jeu d'Apprentissage/Jeu Épistémique, développé dans la théorie de l'action conjointe en didactique (TACD). L'enjeu est de saisir certaines des déterminations des gestes de l'aide ordinaire, lorsque celle-ci se manifeste dans le cours de la classe de mathématiques. Nous observons des gestes d'enseignement stéréotypés qui pourraient s'apparenter à une certaine forme de doxa inhérente à l'aide et qui consisteraient à mettre les élèves en groupe « homogène faible ", à simplifier, voire éparpiller les tâches, à faire réussir les élèves à tout prix au travers de la production de traces, en partie déconnectées des enjeux de savoir. Par ailleurs, l'étude de ces deux situations d'aide ordinaire qui se soldent chacune par un affaiblissement marqué de l'enjeu didactique initial, nous a amenées à caractériser ce que pourraient être des cas didactiques limite, c'est-à-dire des situations de classe où l'on observe de manière liée, un évanouissement des savoirs et des effets de contrat, tels les effets Topaze et/ou Jourdain. Un retour sur l'épistémologie pratique des deux professeurs étudiés nous a permis de saisir certaines des déterminations qui pèsent sur les enseignants dès lors qu'ils décident d'aborder les difficultés d'apprentissage.

This work has been carried out within the framework of the comparatist approach in didactics, and uses the descriptive system of Learning Game/Epistemic game, that was devised in the Joint Action Theory in Didactics. We aim to grasp what could determine the way pedagogical aid is usually provided, when this takes place in a maths class.

We observe two teachers acting in a stereotyped way, which could be seen as a form of doxa constitutive of the methods of pedagogical support. This doxa consists in putting the pupils in «homogenously weak» groups. In these groups, teachers try to simplify or even to fragment tasks, to enable students to succeed at all costs by getting them to produce written records which are partly disconnected from the learning objectives. In fine, re-considering the practical epistemology of the two teachers studied, enable us to capture some of the determinations that affect them, when they decide to address the learning difficulties.

Moreover, the study of these two situations, which lead to the realization a much lower learning objective than initially planned, has enabled us to characterize what could be called « borderline cases ", in other words, the classroom situations where we can observe both disappearance of learning (and of teaching) and appearance of some effects of didactic contract.

\section{INDEX}

Mots-clés : approche comparatiste, jeux d'apprentissage, épistémologie pratique, détermination des gestes de l'aide, cas didactiques limite

Keywords : Joint Action Theory in Didactic (TACD), learning game, practical epistemology, the pedagogical support, teaching and learning in mathematics 
AUTEURS

CORINNE MARLOT

(Université Clermont-Ferrand 2-IUFM d'Auvergne, ACTE-PAEDI)

MARIE TOULLEC-THÉRY

(Université de Nantes-IUFM Pays de la Loire, CREN) 